\title{
From high oscillation to rapid approximation V: The equilateral triangle
}

\author{
Daan Huybrechs* \\ Department of Computer Science \\ Celestijnenlaan 200A \\ B-3001 Leuven \\ Belgium \\ Arieh Iserles \\ DAMTP, Centre for Mathematical Sciences \\ University of Cambridge \\ Wilberforce Rd, Cambridge CB3 0WA \\ United Kingdom \\ Syvert P. Nørsett \\ Department of Mathematics \\ Norwegian University of Science and Technology \\ Trondheim N-7491 \\ Norway
}

November 30, 2009

\begin{abstract}
We address in this paper the approximation of functions in an equilateral triangle by a linear combination of Laplace-Neumann eigenfunctions. The Laplace-Neumann basis exhibits a number of advantages. Thus, the approximations converge fairly fast and their speed of convergence can be much improved by using techniques familiar in Fourier analysis and spectral methods, in particular the hyperbolic cross and polynomial subtraction. Moreover, expansion coefficients can be computed rapidly by a mixture of asymptotic methods and Birkhoff-Hermite quadratures.
\end{abstract}

\section{Introduction}

In this paper we continue a systematic study of expansions in eigenfunctions of the Laplacian subject to Neumann boundary conditions. Such expansions were explored in (Iserles \& Nørsett 2008) for an interval, with generalizations in (Iserles \& Nørsett 2006) to polyharmonic

* This author is a Postdoctoral Fellow of the Research Foundation - Flanders (FWO). 
operators and in (Iserles \& Nørsett 2007) to multivariate domains with tensor-product structure. Convergence acceleration schemes for these expansions were examined in (Huybrechs, Iserles \& Nørsett 2007). The subject of the current paper is the expansion in Laplacian eigenfunctions on the equilateral triangle. This domain does not have tensor-product structure, yet much of the advantageous properties enjoyed by Laplace-Neumann expansions in the earlier cases continue to hold. On the other hand, new hurdles arise that require further insight and different analysis.

Laplace-Neumann expansions in one dimension closely resemble classical Fourier series, hence we also refer to them as modified Fourier series. On the interval, the only difference is a shift in the argument of the sine functions. Yet, unlike Fourier series, Laplace-Neumann expansions converge pointwise in all points of continuity, inclusive of the endpoints. The convergence rate is typically quadratic in the interior, and linear in the endpoints (Olver 2009). This can be improved to arbitrarily high order, for sufficiently smooth functions, by the judicious application of polynomial subtraction techniques (Huybrechs et al. 2007).

Another interesting property is that the coefficients of Laplace-Neumann expansions are amenable to recent methods for the evaluation of highly oscillatory integrals (Iserles \& Nørsett 2005, Huybrechs \& Vandewalle 2006, Olver 2006). We refer the reader to (Huybrechs \& Olver 2009) for a recent review. The cost of these methods is typically independent of the frequency and their accuracy rapidly improves with increasing frequency. As the coefficients of Laplace-Neumman expansions are represented by increasingly oscillatory integrals, application of the above mentioned methods enables their computation one by one: this means that $m$ coefficients can be computed in just $\mathcal{O}(m)$ operations. A careful asymptotic analysis of the coefficients previously revealed that their evaluation can even be considerably more efficient than that of general oscillatory integrals. This was exemplified for Filon-type quadrature rules using derivatives (Iserles \& Nørsett 2005) in the previous papers. As such, the difficulty in constructing modified Fourier series lies mostly in the computation of the first few coefficients, corresponding to non-oscillatory or slowly oscillatory integrals. Though any classical integration scheme may be used to compute the early coefficients, without destroying the $\mathcal{O}(m)$ property, it seems wasteful to igore information that is being used in the evaluation of the later coefficients. In particular, methods for highly oscillatory integrals typically, and unavoidably, require derivatives of the integrand or their approximation at certain critical points. For this reason, research in previous papers focused on the reuse of this information for the evaluation of non-oscillatory integrals. This has led to a variant of the familiar BirkhoffHermite quadrature (Nikolov 1989) which, for lack of an established name, we have termed exotic quadrature.

Rapidly converging series may be obviously truncated for the purpose of finite-precisison computations. This operation is straightforward in one dimension. In more than one dimension, the significant coefficients of Laplace-Neumann expansions exhibit the pattern of the hyperbolic cross. This well-known pattern is seen in various other applications, including multivariate Fourier series (Temlyakov 1993). In general multivariate approximation schemes, using $N$ degrees of freedom per dimension in $d$ dimensions leads to $N^{d}$ coefficients in total. This exponential growth is usually referred to as the curse of dimensionality. The number of coefficients in the hyperbolic cross however grows at a surprisingly slow rate with increasing dimension: it is typically just $\mathcal{O}\left(N(\log N)^{d-1}\right)$, for a comparable accuracy. Exploiting the hyperbolic cross may lead to tremendous computational savings in applications.

Several competitive schemes exist for univariate approximation, such as Chebyshev ex- 
pansions or wavelets (see, e.g., (Boyd 2001) and (Daubechies 1992)), each with advantages and disadvantages. In our opinon, a compelling advantage of modified Fourier series is their natural generalization to triangular domains. This is the topic of the current paper. We intend to generalize the properties of Laplace-Neumann expansions mentioned above to expansions on the equilateral triangle. Eigenfunctions of the Laplacian subject to Neumann (or Dirichlet, for that matter) boundary conditions are known to be a linear combination of plane waves for three types of triangles with special symmetry properties: the equilateral triangle, the right isosceles triangle and the triangle with angles $\pi / 2, \pi / 3$ and $\pi / 6$. Since any triangle can be mapped affinely to any other triangle, it suffices for the purpose of approximation to study a single 'model triangle'. The equilateral triangle exhibits maximal symmetry and, for no other reason, this is the one we study.

We commence our analysis in $\S 2$ with a discussion of Laplace-Neumann eigenfunctions and the associated modified Fourier series on the equilateral triangle, introducing notation and explicit formulæ. At the heart of the theory lies the asymptotic analysis of LaplaceNeumann coefficients in $\S 3$. Based on the information in the asymptotic expansions, the structure of the hyperbolic cross and schemes for convergence acceleration are studied in $\S 4$. Next, we discuss the efficient computation of the coefficients in $\S 5$, with an emphasis on the construction of exotic cubature methods: cubature rules using derivatives for the triangle, which may be interesting in their own right.

\section{Laplace-Neumann eigenfunctions in a triangle}

The eigenfunctions of the Laplacian on a domain $\Omega$ subject to Neumann boundary conditions on the boundary $\partial \Omega$ satisfy

$$
\begin{aligned}
-\Delta u & =\lambda u, \quad x \in \Omega, \\
\frac{\partial u}{\partial n} & =0, \quad x \in \partial \Omega .
\end{aligned}
$$

They were constructed for the equilateral triangle already by Lamé (1833) and presented in different equivalent forms by many authors. We found the construction of Práger (1998) as the most suitable for our ends. That reference presented explicit formulas for an equilateral triangle $\tilde{T}$ with vertices at $\left(-\frac{1}{\sqrt{3}}, 0\right),\left(\frac{1}{\sqrt{3}}, 0\right)$ and $(0,1)$. The eigenfunctions consist of the odd functions (odd with respect to the $y$-axis),

$$
\begin{aligned}
& \tilde{u}_{m, n}(x, y)= 2 \sin \frac{m \pi x}{\sqrt{3}} \cos n \pi y \\
&-2(-1)^{(m+n) / 2} \sin \frac{\pi x}{2 \sqrt{3}}(m+3 n) \cos \frac{\pi y}{2}(m-n) \\
&-2(-1)^{(m-n) / 2} \sin \frac{\pi x}{2 \sqrt{3}}(m-3 n) \cos \frac{\pi y}{2}(m+n), \\
& \quad m, n=1,2, \ldots, \quad m \equiv n \bmod 2, \quad 0<m \leq n,
\end{aligned}
$$

and the even functions

$$
\tilde{v}_{m, n}(x, y)=2 \cos \frac{m \pi x}{\sqrt{3}} \cos n \pi y
$$




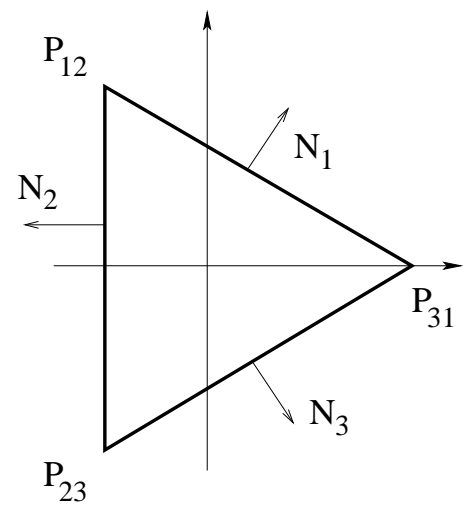

Figure 2.1: Definition of the equilateral triangle $T$. The faces are numbered 1, 2,3 counterclockwise, starting with the upper diagonal face. The vertices are $P_{12}=\left(-\frac{1}{2}, \frac{\sqrt{3}}{2}\right), P_{23}=$ $\left(-\frac{1}{2},-\frac{\sqrt{3}}{2}\right)$ and $P_{31}=(1,0)$.

$$
\begin{gathered}
-2(-1)^{(m+n) / 2} \cos \frac{\pi x}{2 \sqrt{3}}(m+3 n) \cos \frac{\pi y}{2}(m-n) \\
-2(-1)^{(m-n) / 2} \cos \frac{\pi x}{2 \sqrt{3}}(m-3 n) \cos \frac{\pi y}{2}(m+n), \\
m, n=0,1, \ldots, \quad m \equiv n \bmod 2, \quad 0 \leq m \leq n .
\end{gathered}
$$

The corresponding eigenvalues are $\pi^{2}\left(m^{2} / 3+n^{2}\right)$.

We found it more convenient to work with the equilateral triangle $T$ with vertices at $\left(-\frac{1}{2}, \frac{\sqrt{3}}{2}\right),\left(-\frac{1}{2},-\frac{\sqrt{3}}{2}\right)$ and $(1,0)$ as shown in Figure 2.1. This triangle is centred at the origin $(0,0)$ and therefore has simple expressions for all six symmetries of the dihedral group $D_{3}$. This simplies expressions considerably. With some minor additional changes in notation, we write the Laplace-Neumann eigenfunctions as

$$
\begin{array}{ll}
u_{m, n}(x, y)=\operatorname{Im} \psi_{m, n}(x, y), & m=1,2, \ldots, \quad n=0,1, \ldots, \\
v_{m, n}(x, y)=\operatorname{Re} \psi_{m, n}(x, y), \quad m, n=0,1, \ldots,
\end{array}
$$

with the complex-valued function $\psi_{m, n}$ given by

$$
\begin{aligned}
\psi_{m, n}(x, y)= & v_{m, n}(x, y)+\mathrm{i} u_{m, n}(x, y) \\
= & \exp \left(-\frac{2 \pi \mathrm{i} m y}{3 \sqrt{3}}-\frac{\pi \mathrm{i}(m+2 n)(2 x+1)}{3}\right) \\
& +\exp \left(-\frac{2 \pi \mathrm{i} m y}{3 \sqrt{3}}+\frac{\pi \mathrm{i}(m+2 n)(2 x+1)}{3}\right) \\
& +(-1)^{n+m} \exp \left(\frac{2 \pi \mathrm{i}(2 m+3 n) y}{3 \sqrt{3}}-\frac{\pi \mathrm{i} n(2 x+1)}{3}\right) \\
& +(-1)^{n+m} \exp \left(\frac{2 \pi \mathrm{i}(2 m+3 n) y}{3 \sqrt{3}}+\frac{\pi \mathrm{i} n(2 x+1)}{3}\right)
\end{aligned}
$$




$$
\begin{aligned}
& +(-1)^{n} \exp \left(-\frac{2 \pi \mathrm{i}(m+3 n) y}{3 \sqrt{3}}-\frac{\pi \mathrm{i}(m+n)(2 x+1)}{3}\right) \\
& +(-1)^{n} \exp \left(-\frac{2 \pi \mathrm{i}(m+3 n) y}{3 \sqrt{3}}+\frac{\pi \mathrm{i}(m+n)(2 x+1)}{3}\right) .
\end{aligned}
$$

The eigenfunctions of the Laplacian on the equilateral triangle are a sum of six plane waves. The functions $u_{m, n}$ and $v_{m, n}$ correspond to $\tilde{u}_{m, m+2 n}$ and $\tilde{v}_{m, m+2 n}$ as defined above, up to a linear transformation between the triangles $\tilde{T}$ and $T$. The eigenvalues are

$$
\lambda_{m, n}=\frac{16}{27} \pi^{2}\left(m^{2}+3 m n+3 n^{2}\right) .
$$

Figure 2.2 displays a selection of eigenfunctions $u_{m, n}$ and $v_{m, n}$. Note for future reference their increasingly oscillatory character as $n$ grows. It is evident that a great deal of further structure is present: for example, $u_{0,4}$ exhibits symmetry with respect to rotations by $2 \pi / 3$ radians, as is evident from the explicit (and mildly massaged) formula

$$
\begin{aligned}
u_{0,4}(x, y)=1 & -4 \sqrt{3} \sin \frac{8 \pi x}{3} \sin \frac{4 \pi(x+\sqrt{3} y)}{3} \sin \frac{4 \pi(x-\sqrt{3} y)}{3} \\
& -4 \cos \frac{8 \pi x}{3} \cos \frac{4 \pi(x+\sqrt{3} y)}{3} \cos \frac{4 \pi(x-\sqrt{3} y)}{3} .
\end{aligned}
$$

We dwell no further on this issue.

A real-valued function $f(x, y)$ on $T$ can be expanded in the series

$$
f(x, y)=\sum_{m=0}^{\infty} \sum_{n=0}^{\infty} c_{m, n} \frac{v_{m, n}(x, y)}{\left\|v_{m, n}\right\|}+\sum_{m=1}^{\infty} \sum_{n=0}^{\infty} s_{m, n} \frac{u_{m, n}(x, y)}{\left\|u_{m, n}\right\|},
$$

with real expansion coefficients

$$
c_{m, n}=\operatorname{Re}\left\langle f, \psi_{m, n}\right\rangle, \quad \text { and } \quad s_{m, n}=\operatorname{Im}\left\langle f, \psi_{m, n}\right\rangle,
$$

where

$$
\left\langle f, \psi_{m, n}\right\rangle=\hat{f}_{m, n}=\int_{T} f(\boldsymbol{x}) \psi_{m, n}(\boldsymbol{x}) \mathrm{d} V .
$$

The norms of the basis functions are

$$
\left\|v_{m, n}\right\|= \begin{cases}27 \sqrt{3}, & \text { if } m=n=0 \\ \frac{9}{2} \sqrt{3}, & \text { if } m=0 \text { and } n>0 \\ \frac{9}{2} \sqrt{3}, & \text { if } m>0 \text { and } n=0 \\ \frac{9}{4} \sqrt{3}, & \text { otherwise, }\end{cases}
$$

and

$$
\left\|u_{m, n}\right\|= \begin{cases}\frac{9}{2} \sqrt{3}, & \text { if } n=0, \\ \frac{9}{4} \sqrt{3}, & \text { otherwise. }\end{cases}
$$

Before moving on to the asymptotics of $\hat{f}_{m, n}$, let us introduce further notation and conventions. We impose positive orientation on $T$. The equations for the faces are

$$
\begin{array}{ll}
P_{12} P_{23}: & x \equiv-\frac{1}{2}, \\
P_{23} P_{31}: & y=-\frac{\sqrt{3}}{3}(1-x), \\
P_{31} P_{12}: & y=\frac{\sqrt{3}}{3}(1-x),
\end{array}
$$



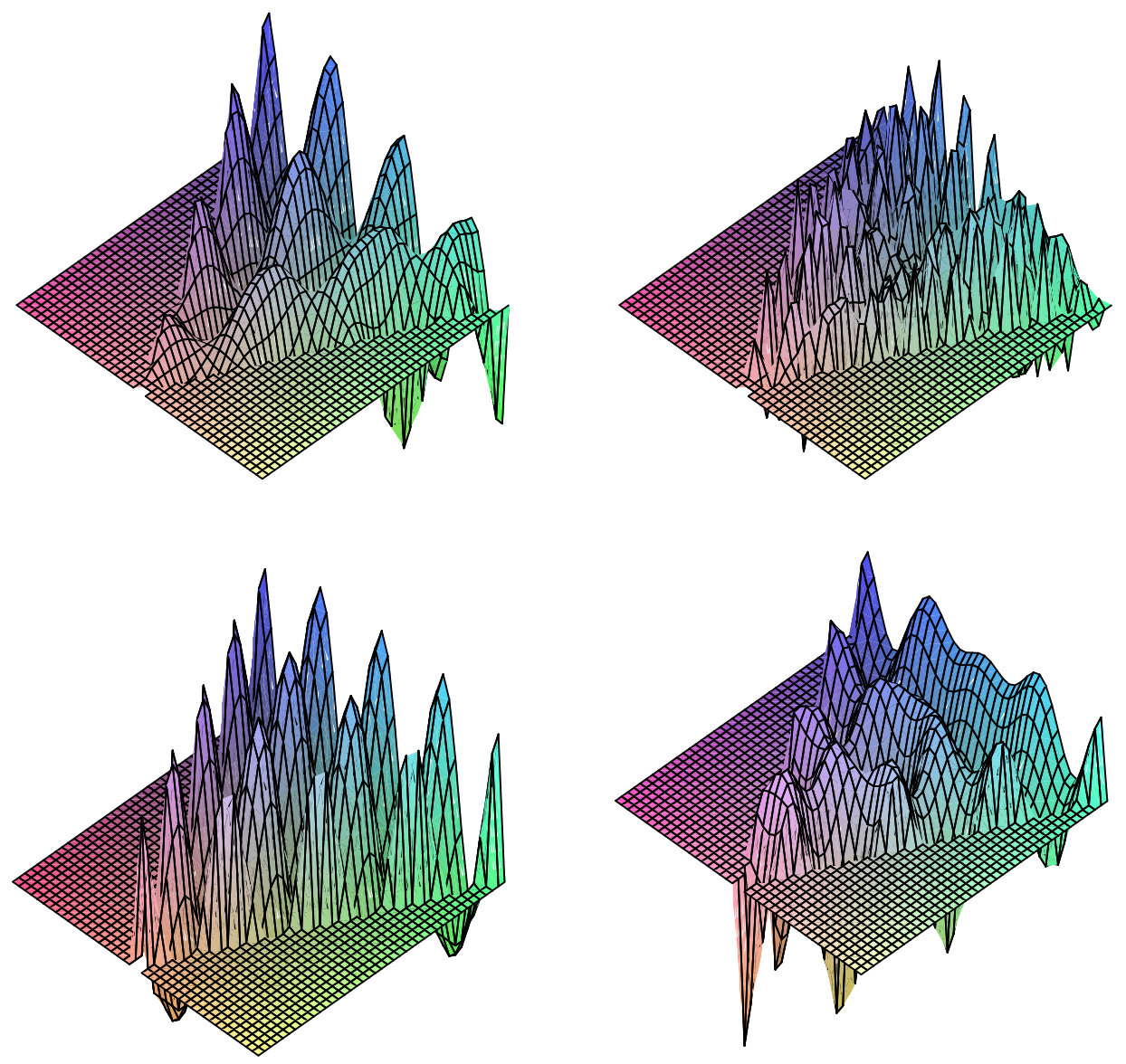

Figure 2.2: The functions $u_{1,3}, u_{3,7}$ (top) and $v_{0,4}, v_{1,3}$ (bottom). Note the trilateral symmetry of $v_{0,4}$ and the oscillatory character of $u_{3,7}$.

the normal derivatives at each of the faces are given by

$$
\begin{aligned}
& N_{1}[f]=\frac{1}{2} f_{x}\left(x, \frac{\sqrt{3}}{3}(1-x)\right)+\frac{\sqrt{3}}{2} f_{y}\left(x, \frac{\sqrt{3}}{3}(1-x)\right), \\
& N_{2}[f]=-f_{x}\left(-\frac{1}{2}, y\right), \\
& N_{3}[f]=\frac{1}{2} f_{x}\left(x,-\frac{\sqrt{3}}{3}(1-x)\right)-\frac{\sqrt{3}}{2} f_{y}\left(x,-\frac{\sqrt{3}}{3}(1-x)\right),
\end{aligned}
$$

and the tangential derivatives by

$$
T_{1}[f]=-\frac{\sqrt{3}}{2} f_{x}\left(x, \frac{\sqrt{3}}{3}(1-x)\right)+\frac{1}{2} f_{y}\left(x, \frac{\sqrt{3}}{3}(1-x)\right),
$$




$$
\begin{aligned}
& T_{2}[f]=-f_{y}\left(-\frac{1}{2}, y\right), \\
& T_{3}[f]=\frac{\sqrt{3}}{2} f_{x}\left(x,-\frac{\sqrt{3}}{3}(1-x)\right)+\frac{1}{2} f_{y}\left(x,-\frac{\sqrt{3}}{3}(1-x)\right) .
\end{aligned}
$$

\section{Asymptotic expansions}

Much information concerning the Laplace-Neumann expansion of a function $f$ is derived from studying the asymptotic behaviour of the coefficients $\hat{f}_{m, n}$. The asymptotic expansion of the coefficients (2.5) for large values of the parameters $m$ and $n$ is the key both to their efficient computation and to convergence acceleration of the series (2.4).

The asymptotic expansion of $\hat{f}_{m, n}$ is rather different from that of the univariate coefficients in (Iserles \& Nørsett 2008) and their generalization to higher-dimensional cubes in (Iserles \& Nørsett 2007), due in part to the non tensor-product shape of the domain and in part to the presence of two parameters $m$ and $n$ that may be small or large. The different asymptotic regimes reduce in our notational framework to just two categories: large $m$ and small $m$. Loosely speaking, large $m$ represents oscillation in both directions of $x$ and $y$ regardless of the value of $n$, whereas small $m$ represent possible oscillation in one direction only, provided $n$ is large. We refer to coefficients in the latter regime as 'edge coefficients' and treat the former case first.

\section{1 $\quad$ Large $m$ asymptotics}

Asymptotic expansions of univariate oscillatory integrals are readily obtained using integration by parts. Its counterpart in higher dimensions is the Stokes theorem. We recall from (Iserles \& Nørsett 2007) the general expansion

$$
\langle f, \psi\rangle \sim-\sum_{k=0}^{\infty} \frac{1}{(-\lambda)^{k+1}} \int_{\partial T} \frac{\partial \Delta^{k} f(\boldsymbol{x})}{\partial n} \psi(\boldsymbol{x}) \mathrm{d} S, \quad \lambda \gg 1
$$

This expansion can be obtained by applying the Stokes theorem twice on the integral (2.5), noting that $\psi(\boldsymbol{x})=-\frac{1}{\lambda} \Delta \psi(\boldsymbol{x})$ :

$$
\begin{aligned}
\langle f, \psi\rangle & =-\frac{1}{\lambda} \int_{T} f(\boldsymbol{x}) \Delta \psi(\boldsymbol{x}) \mathrm{d} V=-\frac{1}{\lambda} \int_{\partial T} f(\boldsymbol{x}) \frac{\partial \psi(\boldsymbol{x})}{\partial n} \mathrm{~d} S+\frac{1}{\lambda} \int_{\partial T} \nabla f(\boldsymbol{x}) \nabla \psi(\boldsymbol{x}) \mathrm{d} S \\
& =-\frac{1}{\lambda} \int_{\partial T} f(\boldsymbol{x}) \frac{\partial \psi(\boldsymbol{x})}{\partial n} \mathrm{~d} S+\frac{1}{\lambda} \int_{\partial T} \frac{\partial f(\boldsymbol{x})}{\partial n} \psi(\boldsymbol{x}) \mathrm{d} S-\frac{1}{\lambda} \int_{T} \Delta f(\boldsymbol{x}) \psi(\boldsymbol{x}) \mathrm{d} V
\end{aligned}
$$

Note that the first term in this expression vanishes due to the Neumann boundary conditions, and therefore

$$
\langle f, \psi\rangle=\frac{1}{\lambda} \int_{\partial T} \frac{\partial f(\boldsymbol{x})}{\partial n} \psi(\boldsymbol{x}) \mathrm{d} S-\frac{1}{\lambda}\langle\Delta f, \psi\rangle .
$$

Iterating the procedure on the latter term ultimately yields (3.1).

Expansion (3.1) is our point of departure. We will obtain the full expansion of the coefficient $\hat{f}_{m, n}$ for large $m$ by expanding each term in (3.1) further. To that end, denote the faces 
of the triangle by $J, K$ and $L$ respectively and define the operators

$$
\begin{aligned}
J_{m, n}[g] & =\int_{J} g(\boldsymbol{x}) \psi_{m, n}(\boldsymbol{x}) \mathrm{d} S, \\
K_{m, n}[g] & =\int_{K} g(\boldsymbol{x}) \psi_{m, n}(\boldsymbol{x}) \mathrm{d} S, \\
L_{m, n}[g] & =\int_{L} g(\boldsymbol{x}) \psi_{m, n}(\boldsymbol{x}) \mathrm{d} S,
\end{aligned}
$$

such that $\int_{\partial T} \frac{\partial f}{\partial n}(\boldsymbol{x}) \psi_{m, n}(\boldsymbol{x}) \mathrm{d} S=J_{m, n}\left[\frac{\partial f}{\partial n}\right]+K_{m, n}\left[\frac{\partial f}{\partial n}\right]+L_{m, n}\left[\frac{\partial f}{\partial n}\right]$. Each of the operators $J, K$ and $L$ can be expanded separately.

Lemma 1 We have

$$
\begin{aligned}
J_{m, n}[g] \sim & -2 \sum_{s=0}^{\infty}\left\{\left(-\frac{3 \sqrt{3}}{2 \pi \mathrm{i} m}\right)^{s+1}+\left[\frac{3 \sqrt{3}}{2 \pi \mathrm{i}(2 m+3 n)}\right]^{s+1}\right. \\
+ & {\left.\left[-\frac{3 \sqrt{3}}{2 \pi \mathrm{i}(m+3 n)}\right]^{s+1}\right\}\left[\mathrm{e}^{-\frac{1}{3} \pi \mathrm{i} m} T_{1}^{s}[g]\left(P_{12}\right)-(-1)^{m} T_{1}^{s}[g]\left(P_{31}\right)\right], }
\end{aligned}
$$

for $m \gg 1$.

Proof The starting point is the expansion, for $a \gg 1$,

$$
\begin{aligned}
& \int_{-\frac{\sqrt{3}}{2}}^{\frac{\sqrt{3}}{2}} G(t) \mathrm{e}^{\pi \mathrm{i}(a t+b)} \mathrm{d} t \\
\sim & -\sum_{s=0}^{\infty} \frac{1}{(-\mathrm{i} \pi a)^{s+1}}\left[\mathrm{e}^{\pi \mathrm{i}\left(\frac{\sqrt{3}}{2} a+b\right)} G^{(s)}\left(\frac{\sqrt{3}}{2}\right)-\mathrm{e}^{\pi \mathrm{i}\left(-\frac{\sqrt{3}}{2} a+b\right)} G^{(s)}\left(-\frac{\sqrt{3}}{2}\right)\right],
\end{aligned}
$$

where $g$ is a smooth function. It can be verified using integration by parts. We parameterize the face $J$ by

$$
P_{31} P_{12}: t \rightarrow\left(\frac{1}{4}-\frac{\sqrt{3}}{2} t, \frac{\sqrt{3}}{4}+\frac{1}{2} t\right), \quad t \in\left[-\frac{\sqrt{3}}{2}, \frac{\sqrt{3}}{2}\right] .
$$

Using this parametrisation, the integral $J_{m, n}[g]$ can be written as the sum of six integrals of the form (3.2), since $\psi_{m, n}$ is a sum of six complex exponentials. Identification of the parameters $a$ and $b$ and straightforward algebra lead to the result.

To shed light on the structure of the expansion, let us define the values

$$
\gamma_{m}^{[1]}=\mathrm{e}^{-\frac{1}{3} \pi \mathrm{i} m}, \quad \gamma_{m}^{[2]}=\mathrm{e}^{\frac{1}{3} \pi \mathrm{i} m}, \quad \gamma_{m}^{[3]}=(-1)^{m} .
$$

We further define

$$
\begin{aligned}
& \kappa_{m, n}^{[s]}=\frac{1}{m^{s+1}}+\frac{(-1)^{s+1}}{(2 m+3 n)^{s+1}}+\frac{1}{(m+3 n)^{s+1}}, \\
& \mathcal{R}_{i}^{[s]}[f]=T_{i+1}^{s} N_{i+1}[f]\left(P_{i, i+1}\right)-T_{i}^{s} N_{i}[f]\left(P_{i, i+1}\right),
\end{aligned}
$$


where the index $i=4$ is identified with $i=1$, such that for example $P_{34} \equiv P_{31}$. These definitions allow the following fully symmetric expansion for the boundary integrals.

Lemma 2 We have

$$
\int_{\partial T} \frac{\partial f}{\partial n} \psi_{m, n} \mathrm{~d} S \sim 2 \sum_{s=0}^{\infty}\left(-\frac{3 \sqrt{3}}{2 \pi \mathrm{i}}\right)^{s+1} \kappa_{m, n}^{[s]} \sum_{i=1}^{3} \gamma_{m}^{[i]} \mathcal{R}_{i}^{[s]}[f],
$$

for $m \gg 1$.

Proof Following Lemma 1, the integral $J_{m, n}\left[\frac{\partial f}{\partial n}\right]$ has the expansion

$$
J_{m, n}\left[\frac{\partial f}{\partial n}\right] \sim-2 \sum_{s=0}^{\infty}\left(-\frac{3 \sqrt{3}}{2 \pi \mathrm{i}}\right)^{s+1} \kappa_{m, n}^{[s]}\left[\mathrm{e}^{-\frac{1}{3} \pi \mathrm{i} m} T_{1}^{s} N_{1}[f]\left(P_{12}\right)-(-1)^{m} T_{1}^{s} N_{1}[f]\left(P_{31}\right)\right],
$$

for $m \gg 1$. With a similar reasoning as in the proof of Lemma 1 we find

$$
\begin{aligned}
& K_{m, n}\left[\frac{\partial f}{\partial n}\right] \sim-2 \sum_{s=0}^{\infty}\left(-\frac{3 \sqrt{3}}{2 \pi \mathrm{i}}\right)^{s+1} \kappa_{m, n}^{[s]}\left[\mathrm{e}^{\frac{1}{3} \pi \mathrm{i} m} T_{2}^{s} N_{2}[f]\left(P_{23}\right)-\mathrm{e}^{-\frac{1}{3} \pi \mathrm{i} m} T_{2}^{s} N_{2}[f]\left(P_{12}\right)\right], \\
& L_{m, n}\left[\frac{\partial f}{\partial n}\right] \sim-2 \sum_{s=0}^{\infty}\left(-\frac{3 \sqrt{3}}{2 \pi \mathrm{i}}\right)^{s+1} \kappa_{m, n}^{[s]}\left[(-1)^{m} T_{3}^{s} N_{3}[f]\left(P_{31}\right)-\mathrm{e}^{\frac{1}{3} \pi \mathrm{i} m} T_{3}^{s} N_{3}[f]\left(P_{23}\right)\right] .
\end{aligned}
$$

Summing these expansions and substituting the definitions of $\gamma_{m}^{[i]}$ and $\mathcal{R}_{i}^{[s]}$ leads to the result.

Note that $(3 \sqrt{3}) /(2 \pi) \approx 0.826993$ and its powers decay fairly gently. It is the decay of $\kappa_{m, n}^{[s]}$ as $m, n, s$ grow that drives convergence of the asymptotic series.

We are ready to assemble results and establish the full asymptotic expansion of the coefficients $\left\langle f, \psi_{m, n}\right\rangle$ for large $m$. We note that this is not an expansion in the Poincaré sense and therefore it is not necessarily unique. It does however carry all necessary information for subsequent developments.

Theorem 3 Let $\hat{f}_{m, n}=c_{m, n}+\mathrm{i} s_{m, n}$ be given by (2.5). It is true for $m \gg 1$ that

$$
\begin{aligned}
c_{m, n} & \sim 2 \sin \frac{\pi m}{3} \sum_{k=0}^{\infty} \sum_{s=0}^{k}\left(\frac{3 \sqrt{3}}{2 \pi}\right)^{2 s+1} \frac{(-1)^{k} \kappa_{m, n}^{[2 s]}}{\lambda_{m, n}^{k-s+1}}\left(\mathcal{R}_{1}^{[2 s]}\left[\Delta^{k-s} f\right]\right. \\
& \left.-\mathcal{R}_{2}^{[2 s]}\left[\Delta^{k-s} f\right]\right)-2 \sum_{k=0}^{\infty} \sum_{s=0}^{k}\left(\frac{3 \sqrt{3}}{2 \pi}\right)^{2 s+2} \frac{(-1)^{k} \kappa_{m, n}^{[2 s+1]}}{\lambda_{m, n}^{k-s+1}} \\
& \times\left\{\cos \frac{\pi m}{3}\left(\mathcal{R}_{1}^{[2 s+1]}\left[\Delta^{k-s} f\right]+\mathcal{R}_{2}^{[2 s+1]}\left[\Delta^{k-s} f\right]\right)+(-1)^{m} \mathcal{R}_{3}^{[2 s+1]}\left[\Delta^{k-s} f\right]\right\}
\end{aligned}
$$

and

$$
s_{m, n} \sim 2 \sum_{k=0}^{\infty} \sum_{s=0}^{k}\left(\frac{3 \sqrt{3}}{2 \pi}\right)^{2 s+1} \frac{(-1)^{k} \kappa_{m, n}^{[2 s]}}{\lambda_{m, n}^{k-s+1}}\left\{\operatorname { c o s } \frac { \pi m } { 3 } \left(\mathcal{R}_{1}^{[2 s]}\left[\Delta^{k-s} f\right]\right.\right.
$$




$$
\begin{aligned}
& \left.\left.+\mathcal{R}_{2}^{[2 s]}\left[\Delta^{k-s} f\right]\right)+(-1)^{m} \mathcal{R}_{3}^{[2 s]}\left[\Delta^{k-s} f\right]\right\} \\
& +2 \sin \frac{\pi m}{3} \sum_{k=0}^{\infty} \sum_{s=0}^{k}\left(\frac{3 \sqrt{3}}{2 \pi}\right)^{2 s+2} \frac{(-1)^{k} \kappa_{m, n}^{[2 s+1]}}{\lambda_{m, n}^{k-s+1}}\left(\mathcal{R}_{1}^{[2 s+1]}\left[\Delta^{k-s} f\right]\right. \\
& \left.-\mathcal{R}_{2}^{[2 s+1]}\left[\Delta^{k-s} f\right]\right)
\end{aligned}
$$

Proof From (3.1) and Lemma 2 we have

$$
\begin{aligned}
\hat{f}_{m, n} \sim & -2 \sum_{k=0}^{\infty} \frac{(-1)^{k+1}}{\lambda_{m, n}^{k+1}} \sum_{s=0}^{\infty}\left(-\frac{3 \sqrt{3}}{2 \pi \mathrm{i}}\right)^{s+1} \kappa_{m, n}^{[s]} \sum_{i=1}^{3} \gamma_{m}^{[i]} \mathcal{R}_{i}^{[s]}\left[\Delta^{k} f\right] \\
= & -2 \sum_{s=0}^{\infty}\left(-\frac{3 \sqrt{3}}{2 \pi \mathrm{i}}\right)^{2 s+1} \sum_{k=s}^{\infty} \frac{(-1)^{k-s+1}}{\lambda_{m, n}^{k-s+1}} \kappa_{m, n}^{[2 s]} \sum_{i=1}^{3} \gamma_{m}^{[i]} \mathcal{R}_{i}^{[2 s]}\left[\Delta^{k-s} f\right] \\
& -2 \sum_{s=0}^{\infty}\left(-\frac{3 \sqrt{3}}{2 \pi \mathrm{i}}\right)^{2 s+2} \sum_{k=s}^{\infty} \frac{(-1)^{k-s+1}}{\lambda_{m, n}^{k-s+1}} \kappa_{m, n}^{[2 s+1]} \sum_{i=1}^{3} \gamma_{m}^{[i]} \mathcal{R}_{i}^{[2 s+1]}\left[\Delta^{k-s} f\right] \\
= & 2 \mathrm{i} \sum_{k=0}^{\infty} \sum_{s=0}^{k}\left(\frac{3 \sqrt{3}}{2 \pi}\right)^{2 s+1} \frac{(-1)^{k}}{\lambda_{m, n}^{k-s+1}} \kappa_{m, n}^{[2 s]} \sum_{i=1}^{3} \gamma_{m}^{[i]} \mathcal{R}_{i}^{[2 s]}\left[\Delta^{k-s} f\right] \\
& -2 \sum_{k=0}^{\infty} \sum_{s=0}^{k}\left(\frac{3 \sqrt{3}}{2 \pi}\right)^{2 s+2} \frac{(-1)^{k}}{\lambda_{m, n}^{k-s+1}} \kappa_{m, n}^{[2 s+1]} \sum_{i=1}^{3} \gamma_{m}^{[i]} \mathcal{R}_{i}^{[2 s+1]}\left[\Delta^{k-s} f\right] .
\end{aligned}
$$

The result follows by taking the real and imaginary parts of this expression.

Note that we have aggregated terms to be of an equal order of magnitude for $m \gg 1$, bearing in mind that

$$
\frac{1}{\lambda_{m, n}^{k+1}} \sim \mathcal{O}\left(m^{-2 k-2}\right), \quad \kappa_{m, n}^{[s]} \sim \mathcal{O}\left(m^{-s-1}\right)
$$

where $\lambda_{m, n}$ has been defined in (2.3). It follows at once that truncating the outer summation in (3.4) or (3.5) after $L$ terms yields an asymptotic approximation that carries an error of size $\mathcal{O}\left(m^{-2 L-3}\right)$ for $m \gg 1$.

The expressions can be further simplified by noting that everything can be reduced to the calculation of partial derivatives of $f$ at the vertices. Indeed one can verify that $\mathcal{R}_{i}^{[s]}[f]$ can, for example, be written in terms of tangential derivatives as

$$
\mathcal{R}_{i}^{[s]}[f]=\frac{1}{\sqrt{3}}\left(T_{i}^{s+1}+2 T_{i}^{s} T_{i+1}+2 T_{i} T_{i+1}^{s}+T_{i+1}^{s+1}\right) f\left(P_{i, i+1}\right) .
$$

Likewise, we can write

$$
\Delta=\frac{4}{3}\left(T_{1}^{2}+T_{1} T_{2}+T_{2}^{2}\right)=\frac{4}{3}\left(T_{2}^{2}+T_{2} T_{3}+T_{3}^{2}\right)=\frac{4}{3}\left(T_{3}^{2}+T_{3} T_{1}+T_{1}^{2}\right) .
$$


Together this leads to the explicit expression

$$
\mathcal{R}_{1}^{[2 s]}\left[\Delta^{k-s} f\right]=\left(\frac{4}{3}\right)^{k-s} \frac{1}{\sqrt{3}}\left(T_{1}^{s+1}+2 T_{1}^{s} T_{2}+2 T_{1} T_{2}^{s}+T_{2}^{s+1}\right)\left(T_{1}^{2}+T_{1} T_{2}+T_{2}^{2}\right)^{k-s} f\left(P_{1,2}\right)
$$

and to similar explicit expressions for the other terms of this form.

\subsection{Edge coefficients}

The asymptotic expansion of the coefficients for $m \gg 1$ depends only on partial derivatives of $f$ at the vertices. This result was obtained in two steps: the Stokes theorem pushes the integral to the boundary, partial integration of the boundary terms next pushes the integral further to the vertices. In the case of edge coefficients, where $m \sim 1$ and $n \gg 1$, the first step remains possible but the second requires alteration.

To be precise, we note that expansion (3.1) remains valid because $\lambda_{m, n} \gg 1$ if $n \gg 1$. Let us state the counterpart of Lemma 1. To that end we define the function

$$
\Psi_{m}(t):=\mathrm{e}^{\frac{2}{9} \sqrt{3} \mathrm{i} \pi m t}
$$

Lemma 4 We have

$$
\begin{aligned}
J_{m, n}[g] \sim 2 \mathrm{e}^{-\frac{2}{3} \pi \mathrm{i} m} \int_{-\frac{\sqrt{3}}{2}}^{\frac{\sqrt{3}}{2}} g\left(\frac{1}{4}-\frac{\sqrt{3}}{2} t, \frac{\sqrt{3}}{4}+\frac{1}{2} t\right) \Psi_{m}(t) \mathrm{d} t-2 \sum_{s=0}^{\infty}\left\{\left[\frac{3 \sqrt{3}}{2 \pi \mathrm{i}(2 m+3 n)}\right]^{s+1}\right. \\
\left.+\left[-\frac{3 \sqrt{3}}{2 \pi \mathrm{i}(m+3 n)}\right]^{s+1}\right\}\left[\mathrm{e}^{-\frac{1}{3} \pi \mathrm{i} m} T_{1}^{s}[g]\left(P_{12}\right)-(-1)^{m} T_{1}^{s}[g]\left(P_{31}\right)\right],
\end{aligned}
$$

for $n \gg 1$.

Proof Proceeding exactly as in the proof of Lemma 1, we obtain a sum of six integrals of the form (3.2). Explicit computations show that only four of those can be expanded as before. Two integrals are non-oscillatory and lead to the non-oscillatory integral in the above expansion.

The result contains a non-oscillatory integral along the edge $J$. Similar results can be obtained for the other edges. Let us define the values

$$
\begin{aligned}
& \tilde{\gamma}_{m}^{[1]}=2 \mathrm{e}^{-\frac{2}{3} \pi \mathrm{i} m}, \quad \tilde{\gamma}_{m}^{[2]}=2, \quad \tilde{\gamma}_{m}^{[3]}=2 \mathrm{e}^{\frac{2}{3} \pi \mathrm{i} m} \\
& \tilde{\kappa}_{m, n}^{[s]}=\frac{(-1)^{s+1}}{(2 m+3 n)^{s+1}}+\frac{1}{(m+3 n)^{s+1}}
\end{aligned}
$$

and the functionals

$$
\begin{aligned}
& B_{m}^{[1]}[g]=\int_{-\frac{\sqrt{3}}{2}}^{\frac{\sqrt{3}}{2}} g\left(\frac{1}{4}-\frac{\sqrt{3}}{2} t, \frac{\sqrt{3}}{4}+\frac{1}{2} t\right) \Psi_{m}(t) \mathrm{d} t \\
& B_{m}^{[2]}[g]=\int_{-\frac{\sqrt{3}}{2}}^{\frac{\sqrt{3}}{2}} g\left(-\frac{1}{2},-t\right) \Psi_{m}(t) \mathrm{d} t
\end{aligned}
$$




$$
B_{m}^{[3]}[g]=\int_{-\frac{\sqrt{3}}{2}}^{\frac{\sqrt{3}}{2}} g\left(\frac{1}{4}+\frac{\sqrt{3}}{2} t,-\frac{\sqrt{3}}{4}+\frac{1}{2} t\right) \Psi_{m}(t) \mathrm{d} t .
$$

These functionals correspond to line integrals along the faces $J, K$ and $L$ respectively. We can now state the counterpart of Lemma 2.

Lemma 5 We have

$$
\int_{\partial T} \frac{\partial f}{\partial n} \psi_{m, n} \mathrm{~d} S \sim \sum_{i=1}^{3} \tilde{\gamma}_{m}^{[i]} B_{m}^{[i]} N_{i} f+2 \sum_{s=0}^{\infty}\left(-\frac{3 \sqrt{3}}{2 \pi \mathrm{i}}\right)^{s+1} \tilde{\kappa}_{m, n}^{[s]} \sum_{i=1}^{3} \gamma_{m}^{[i]} \mathcal{R}_{i}^{[s]}[f],
$$

for $n \gg 1$.

The structure of the asymptotic expansion for $n \gg 1$ is very similar to that for $m \gg 1$, with the exception of the appearance of the non-oscillatory boundary integrals $B_{m}^{[i]} N_{i} f$. The appearance of non-oscillatory integrals may not come as a surprise, it was also observed for edge coefficients in the case of modified Fourier expansions in a $d$-dimensional cube in (Iserles \& Nørsett 2007). Here, the non-oscillatory integrals correspond to integrals along the boundary involving the univariate function $\Psi_{m}(t)$.

Theorem 3 can be altered accordingly.

Theorem 6 Let $\hat{f}_{m, n}=c_{m, n}+\mathrm{i} s_{m, n}$ be given by (2.5). It is true for $n \gg 1$ that

$$
\begin{aligned}
c_{m, n} \sim & \sum_{k=0}^{\infty} \frac{(-1)^{k}}{\lambda_{m, n}^{k+1}} \sum_{i=1}^{3} \operatorname{Re}\left(\tilde{\gamma}_{m}^{[i]} B_{m}^{[i]} N_{i} \Delta^{k} f\right)+2 \sin \frac{\pi m}{3} \sum_{k=0}^{\infty} \sum_{s=0}^{k}\left(\frac{3 \sqrt{3}}{2 \pi}\right)^{2 s+1} \frac{(-1)^{k} \tilde{\kappa}_{m, n}^{[2 s]}}{\lambda_{m, n}^{k-s+1}} \\
& \times\left(\mathcal{R}_{1}^{[2 s]}\left[\Delta^{k-s} f\right]-\mathcal{R}_{2}^{[2 s]}\left[\Delta^{k-s} f\right]\right)-2 \sum_{k=0}^{\infty} \sum_{s=0}^{k}\left(\frac{3 \sqrt{3}}{2 \pi}\right)^{2 s+2} \frac{(-1)^{k} \tilde{\kappa}_{m, n}^{[2 s+1]}}{\lambda_{m, n}^{k-s+1}} \\
& \times\left\{\cos \frac{\pi m}{3}\left(\mathcal{R}_{1}^{[2 s+1]}\left[\Delta^{k-s} f\right]+\mathcal{R}_{2}^{[2 s+1]}\left[\Delta^{k-s} f\right]\right)+(-1)^{m} \mathcal{R}_{3}^{[2 s+1]}\left[\Delta^{k-s} f\right]\right\}
\end{aligned}
$$

and

$$
\begin{aligned}
s_{m, n} \sim & \sum_{k=0}^{\infty} \frac{(-1)^{k}}{\lambda_{m, n}^{k+1}} \sum_{i=1}^{3} \operatorname{Im}\left(\tilde{\gamma}_{m}^{[i]} B_{m}^{[i]} N_{i} \Delta^{k} f\right)+2 \sum_{k=0}^{\infty} \sum_{s=0}^{k}\left(\frac{3 \sqrt{3}}{2 \pi}\right)^{2 s+1} \frac{(-1)^{k} \kappa_{m, n}^{[2 s]}}{\lambda_{m, n}^{k-s+1}} \\
& \times\left\{\cos \frac{\pi m}{3}\left(\mathcal{R}_{1}^{[2 s]}\left[\Delta^{k-s} f\right]+\mathcal{R}_{2}^{[2 s]}\left[\Delta^{k-s} f\right]\right)+(-1)^{m} \mathcal{R}_{3}^{[2 s]}\left[\Delta^{k-s} f\right]\right\} \\
& +2 \sin \frac{\pi m}{3} \sum_{k=0}^{\infty} \sum_{s=0}^{k}\left(\frac{3 \sqrt{3}}{2 \pi}\right)^{2 s+2} \frac{(-1)^{k} \kappa_{m, n}^{[2 s+1]}}{\lambda_{m, n}^{k-s+1}}\left(\mathcal{R}_{1}^{[2 s+1]}\left[\Delta^{k-s} f\right]\right. \\
& \left.-\mathcal{R}_{2}^{[2 s+1]}\left[\Delta^{k-s} f\right]\right) .
\end{aligned}
$$



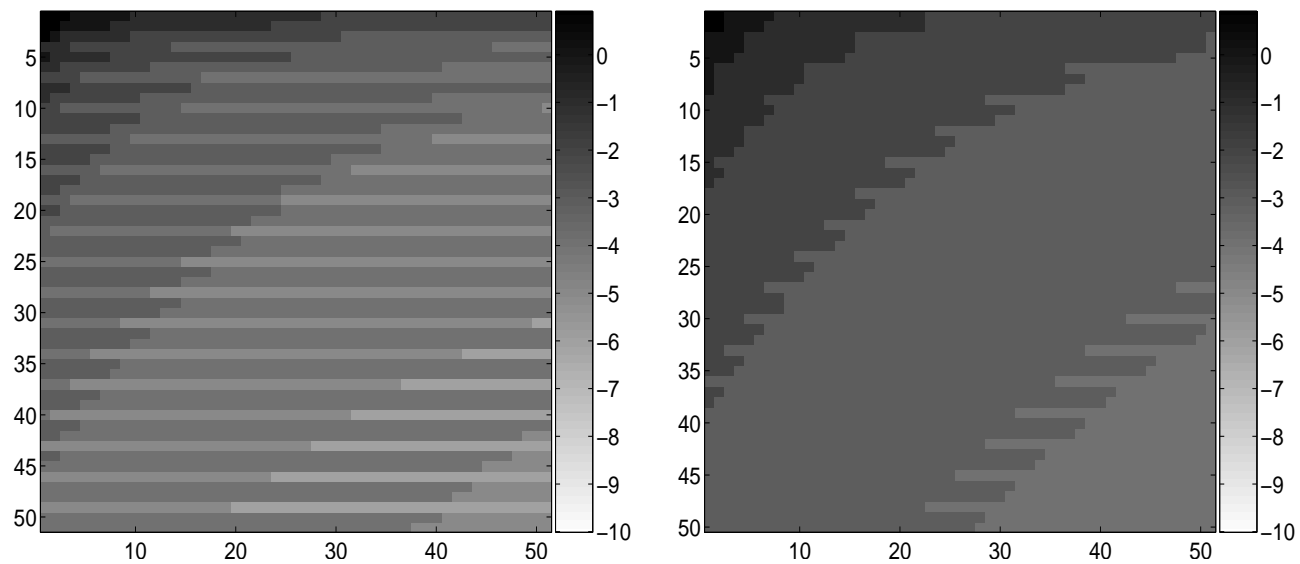

Figure 4.1: Plot of the base-10 logarithm of the size of the coefficients $c_{m, n}$ (left) and $s_{m, n}$ (right) for the function $f(x, y)=\mathrm{e}^{\pi(x-y)}$ in the $(m, n)$ plane. Rows correspond to fixed $m$, columns to fixed $n$.

\section{Hyperbolic cross}

Figure 4.1 displays the size of the coefficients $c_{m, n}$ for the smooth function $f(x, y)=\mathrm{e}^{\pi(x-y)}$. The figure clearly exhibits the hyperbolic cross phenomenon: since the coefficients decay both with increasing $m$ and increasing $n$, the level curves in the figure have hyperbolic shape. Coefficients are largest near the edges, corresponding to small $m$ or small $n$, and they decay most rapidly in the region where both $m$ and $n$ grow large. A small perturbation in the smoothness of the level curves is observed on every third row: coefficients are smaller when $m \equiv 0(\bmod 3)$.

In this section we examine the structure of the hyperbolic cross and we explore the acceleration of convergence by the application of polynomial subtraction.

\subsection{The leading order term}

The structure of this hyperbolic cross can be fully explained from the asymptotic expansions that were derived in the previous section. Looking just at the leading order term in (3.4) and (3.5), we have

$$
\begin{aligned}
c_{m, n} \sim & \frac{3 \sqrt{3} \sin \frac{\pi m}{3}}{\pi} \frac{\kappa_{m, n}^{[0]}}{\lambda_{m, n}}\left(\mathcal{R}_{1}^{[0]}[f]-\mathcal{R}_{2}^{[0]}[f]\right) \\
& -\frac{27}{2 \pi^{2}} \frac{\kappa_{m, n}^{[1]}}{\lambda_{m, n}}\left\{\cos \frac{\pi m}{3}\left(\mathcal{R}_{1}^{[1]}[f]+\mathcal{R}_{2}^{[1]}[f]\right)+(-1)^{m} \mathcal{R}_{3}^{[1]}[f]\right\} \\
= & \frac{729 \sin \frac{\pi m}{3}}{32 \pi^{3}} \frac{1}{m\left(m+\frac{3}{2} n\right)(m+3 n)}\left[\left(T_{1}+T_{2}\right) f\left(P_{1,2}\right)-\left(T_{2}+T_{3}\right) f\left(P_{2,3}\right)\right] \\
& -\frac{2187 \sqrt{3}}{128 \pi^{4}} \frac{m^{2}+3 m n+3 n^{2}}{m^{2}\left(m+\frac{3}{2} n\right)^{2}(m+3 n)^{2}}\left[\cos \frac{\pi m}{3}\left(T_{1}^{2}+4 T_{1} T_{2}+T_{2}^{2}\right) f\left(P_{1,2}\right)\right.
\end{aligned}
$$




$$
\left.+\cos \frac{\pi m}{3}\left(T_{2}^{2}+4 T_{2} T_{3}+T_{3}^{2}\right) f\left(P_{2,3}\right)+(-1)^{m}\left(T_{3}^{2}+4 T_{3} T_{1}+T_{1}^{2}\right) f\left(P_{3,1}\right)\right],
$$

and

$$
\begin{aligned}
s_{m, n} \sim & \frac{729}{32 \pi^{3}} \frac{1}{m\left(m+\frac{3}{2} n\right)(m+3 n)}\left[\cos \frac{\pi m}{3}\left(T_{1}+T_{2}\right) f\left(P_{1,2}\right)\right. \\
& \left.\quad+\cos \frac{\pi m}{3}\left(T_{2}+T_{3}\right) f\left(P_{2,3}\right)+(-1)^{m}\left(T_{3}+T_{1}\right) f\left(P_{3,1}\right)\right] \\
& +\frac{2187 \sqrt{3} \sin \frac{\pi m}{3}}{128 \pi^{4}} \frac{m^{2}+3 m n+3 n^{2}}{m^{2}\left(m+\frac{3}{2} n\right)^{2}(m+3 n)^{2}}\left[\left(T_{1}^{2}+4 T_{1} T_{2}+T_{2}^{2}\right) f\left(P_{1,2}\right)\right. \\
& \left.-\left(T_{2}^{2}+4 T_{2} T_{3}+T_{3}^{2}\right) f\left(P_{2,3}\right)\right] .
\end{aligned}
$$

Therefore, for $m \gg 1$

$$
\begin{aligned}
& c_{m, n} \sim\left\{\begin{array}{lll}
\mathcal{O}\left(m^{-3}\right), & m \neq 0 & (\bmod 3), \\
\mathcal{O}\left(m^{-4}\right), & m=0 & (\bmod 3)
\end{array}\right. \\
& s_{m, n} \sim \mathcal{O}\left(m^{-3}\right) .
\end{aligned}
$$

This explains the ripple every third row in Figure 4.1. The coefficients $c_{m, n}$ decay faster when $m=0(\bmod 3)$ because the first term in the asymptotic expansion vanishes. This feature is not present in the $s_{m, n}$ coefficients.

In the direction of increasing $n$ (from left to right in Figure 4.1) the coefficients decay as $\mathcal{O}\left(n^{-2}\right)$. In general, the shape of the hyperbolic cross is determined by the factor

$$
\frac{1}{m\left(m+\frac{3}{2} n\right)(m+3 n)}
$$

which appears in the leading order term of both $c_{m, n}$ and $s_{m, n}$.

\subsection{Polynomial subtraction at vertices}

The situation changes significantly when first derivatives (and hence also tangential derivatives) of $f$ vanish at the vertices. Then we find from the asymptotic expansion for $m \gg 1$ that

$$
\begin{aligned}
c_{m, n} & \sim \mathcal{O}\left(m^{-4}\right), \\
s_{m, n} & \sim \begin{cases}\mathcal{O}\left(m^{-4}\right), & m \neq 0(\bmod 3), \\
\mathcal{O}\left(m^{-5}\right), & m=0(\bmod 3),\end{cases}
\end{aligned}
$$

(for $s_{m, n}$ and the $m=0(\bmod 3)$ case we need the next term in the expansion but it is equally straightforward to derive). The situation is now reversed: the coefficients $s_{m, n}$ decay faster if $m=0(\bmod 3)$. The coefficients $c_{m, n}$ behave as $\mathcal{O}\left(m^{-4}\right)$ for all $m$.

In either case, both sets of coefficients $c_{m, n}$ and $s_{m, n}$ decay faster when derivatives of $f$ at the vertices vanish. We can exploit this behaviour by constructing a polynomial $p$ that interpolates first derivatives of $f$ at the vertices and expanding the function $\tilde{f}=f-p$. By construction, $\tilde{f}$ yields more rapid decay of its coefficients for $m \gg 1$ than the original function 

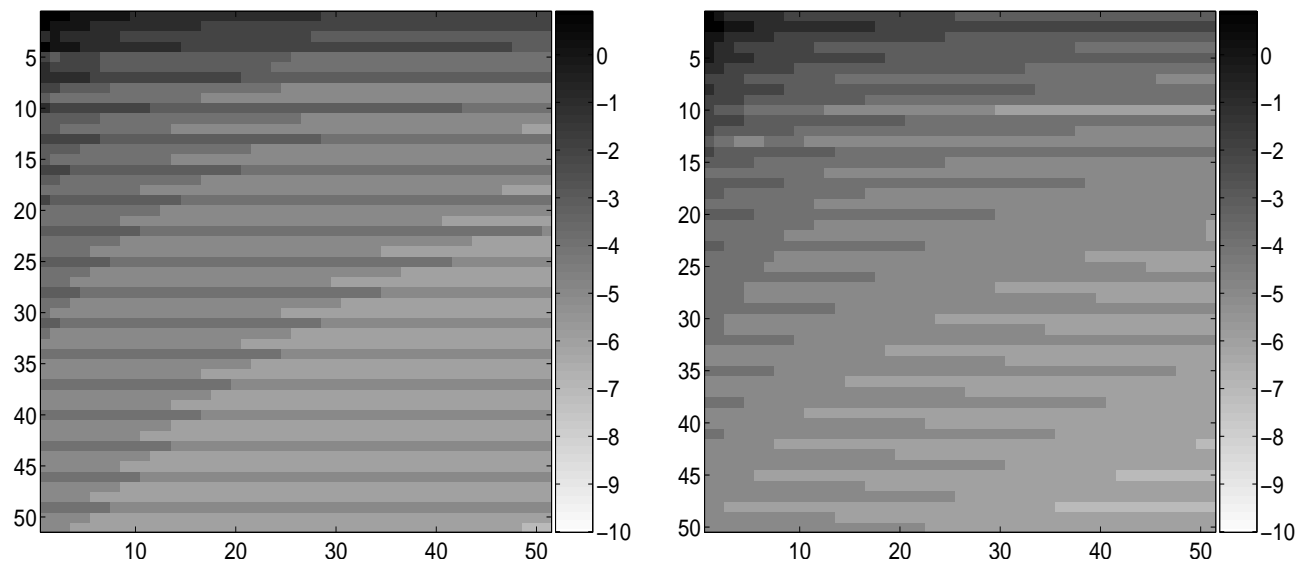

Figure 4.2: Plot of the base-10 logarithm of the size of the coefficients $c_{m, n}$ (left) and $s_{m, n}$ (right) for the function $\mathrm{e}^{\pi(x-y)}-p(x, y)$ in the $(m, n)$ plane.

$f$. This technique was detailed for the $d$-dimensional cube in (Huybrechs et al. 2007) but is equally applicable to the triangle. To be precise, consider a polynomial $p$ such that

$$
\begin{aligned}
& \left(T_{1}+T_{2}\right) p\left(P_{12}\right)=\left(T_{1}+T_{2}\right) f\left(P_{12}\right), \\
& \left(T_{2}+T_{3}\right) p\left(P_{23}\right)=\left(T_{2}+T_{3}\right) f\left(P_{23}\right), \\
& \left(T_{3}+T_{1}\right) p\left(P_{31}\right)=\left(T_{3}+T_{1}\right) f\left(P_{31}\right) .
\end{aligned}
$$

These conditions are obtained from the expressions (4.1) and (4.2) and they guarantee that the leading order terms of the coefficients of $\tilde{f}$ vanish. We find a suitable polynomial of the form $p(x, y)=a x^{2} y+b x y+c y^{2} x$, where the coefficients $a, b$ and $c$ are determined from the conditions (4.4). The expansion coefficients of $\tilde{f}(x, y)=\mathrm{e}^{\pi(x-y)}-p(x, y)$ are plotted in Figure 4.2. Note the accelerated decay of the coefficients for increasing $m$ (from top to bottom).

We may wish to accelerate decay of the coefficients further by cancelling, say, $S \geq 2$ terms in the expansion. This is achieved in full generality by the interpolation conditions

$$
\begin{array}{ll}
\mathcal{R}_{1}^{[s]}[p]=\mathcal{R}_{1}^{[s]}[f], & s=0,1, \ldots, S-1, \\
\mathcal{R}_{2}^{[s]}[p]=\mathcal{R}_{2}^{[s]}[f], & s=0,1, \ldots, S-1, \\
\mathcal{R}_{3}^{[s]}[p]=\mathcal{R}_{3}^{[s]}[f], & s=0,1, \ldots, S-1,
\end{array}
$$

as is evident from Theorem 3. The result for $S=2$ is shown in Figure 4.3. We denoted by $q(x, y)$ the polynomial that satisfies conditions (4.5) for $S=2$.

\subsection{Accelerated decay of edge coefficients}

In order to accelerate the decay of edge coefficients, where $m \sim 1$ and $n \gg 1$, it is not sufficient to interpolate derivatives at the vertices. Compared to the case of large $m$, more 

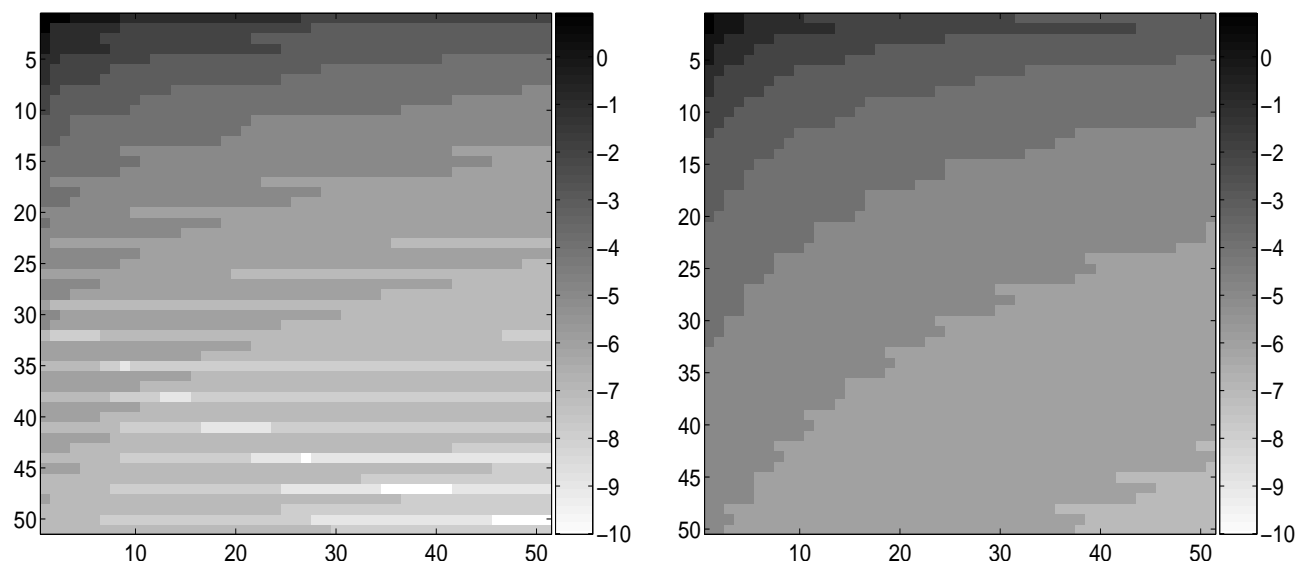

Figure 4.3: Plot of the base-10 logarithm of the size of the coefficients $c_{m, n}$ (left) and $s_{m, n}$ (right) for the function $\mathrm{e}^{\pi(x-y)}-q(x, y)$ in the $(m, n)$ plane.

boundary data has entered the asymptotic expansions as derived in Theorem 6, in the form of the non-oscillatory integrals $B_{m}^{[i]} N_{i} f$. Interpolation of these integrals will accelerate the decay of these coefficients too. As the integrals depend on $m$ however, one would have to interpolate these values for a range of values of $m$, from $m=0$ up to a value that is sufficiently large so that large- $m$ asymptotics kicks in.

Alternatively, it is conceptually and computationally easier to interpolate normal derivatives $N_{i} f$ along the entire boundary. This is sufficient to render the first term of the expansion zero regardless of the value of $m$, as all boundary integrals $B_{m}^{[i]} N_{i} f$ are automatically interpolated as well. The construction of smooth functions interpolating boundary values is a topic investigated in the context of Computer Aided Geometric Design (Farin 1997). Herewith we present a smooth interpolation to first-order normal derivative data. However, before doing so it is instructive to start with the easier case of the interpolation of Dirichlet boundary data.

We identify with $(x, y) \in T$ six points on $\partial T$,

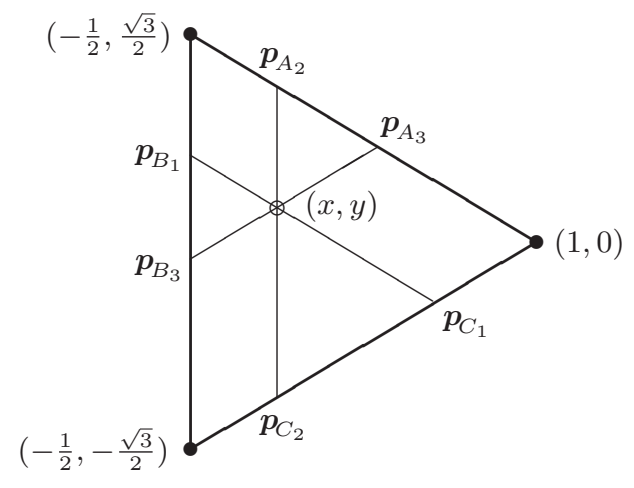

- in other words, the line segment $\left[\boldsymbol{p}_{B_{1}}, \boldsymbol{p}_{C_{1}}\right]$ is parallel to the first face, $\left[\boldsymbol{p}_{A_{2}}, \boldsymbol{p}_{C_{2}}\right]$ to the 
second and $\left[\boldsymbol{p}_{A_{3}}, \boldsymbol{p}_{B_{3}}\right]$ to the third. All of them pass through $(x, y)$. Specifically,

$$
\begin{aligned}
& \boldsymbol{p}_{A_{2}}(x, y)=\left(x, \frac{1}{\sqrt{3}}(1-x)\right), \\
& \boldsymbol{p}_{A_{3}}(x, y)=\left(\frac{1}{2}(1+x-\sqrt{3} y), \frac{1}{2 \sqrt{3}}(1-x+\sqrt{3} y)\right), \\
& \boldsymbol{p}_{B_{1}}(x, y)=\left(-\frac{1}{2}, \frac{1}{\sqrt{3}}\left(\frac{1}{2}+x+\sqrt{3} y\right)\right), \\
& \boldsymbol{p}_{B_{3}}(x, y)=\left(-\frac{1}{2}, \frac{1}{\sqrt{3}}\left(-\frac{1}{2}-x+\sqrt{3} y\right)\right), \\
& \boldsymbol{p}_{C_{1}}(x, y)=\left(\frac{1}{2}(1+x+\sqrt{3} y), \frac{1}{2 \sqrt{3}}(-1+x+\sqrt{3} y)\right), \\
& \boldsymbol{p}_{C_{2}}(x, y)=\left(x,-\frac{1}{\sqrt{3}}(1-x)\right) .
\end{aligned}
$$

Along each line segment we form a linear interpolation to $f$,

$$
\begin{aligned}
& q_{A}(x, y)=\frac{1-x+\sqrt{3} y}{2+x+\sqrt{3} y} f\left(\boldsymbol{p}_{B_{1}}(x, y)\right)+\frac{2 x+1}{2+x+\sqrt{3} y} f\left(\boldsymbol{p}_{C_{1}}(x, y)\right), \\
& q_{B}(x, y)=\frac{1-x+\sqrt{3} y}{2(1-x)} f\left(\boldsymbol{p}_{A_{2}}(x, y)\right)+\frac{1-x-\sqrt{3} y}{2(1-x)} f\left(\boldsymbol{p}_{C_{2}}(x, y)\right), \\
& q_{C}(x, y)=\frac{2 x+1}{2+x-\sqrt{3} y} f\left(\boldsymbol{p}_{A_{3}}(x, y)\right)+\frac{1-x-\sqrt{3} y}{2+x-\sqrt{3} y} f\left(\boldsymbol{p}_{B_{3}}(x, y)\right) .
\end{aligned}
$$

Each of these functions agrees with $f$ on two edges and is linear in $x$ and $y$ along the third edge. The sum of all three functions therefore equals twice the value of $f$ along the edges plus a linear function in $x$ and $y$. It is sufficient to halve the sum and remove the linear function, and to that end we let

$$
\begin{aligned}
q(x, y)= & \frac{1}{2}\left[q_{A}(x, y)+q_{B}(x, y)+q_{C}(x, y)\right]-\frac{1}{6}(2 x+1) f(1,0) \\
& -\frac{1}{6}(1-x+\sqrt{3} y) f\left(-\frac{1}{2}, \frac{\sqrt{3}}{2}\right)-\frac{1}{6}(1-x-\sqrt{3} y) f\left(-\frac{1}{2},-\frac{\sqrt{3}}{2}\right) .
\end{aligned}
$$

Simple algebra affirms that $q=f$ along the boundary of $T$, therefore (4.6) interpolates Dirichlet boundary conditions. The apparent singularity of, for example, $q_{B}$ along the line $x=1$ is removable since along that line we also have $f\left(\boldsymbol{p}_{A_{2}}(1, y)\right)=f\left(\boldsymbol{p}_{C_{2}}(1, y)\right)$. A similar observation holds for the other two functions. Thus the interpolant is a smooth function.

The case of normal derivatives is slightly more involved. We now employ cubic interpolation along the line segments,

$$
\begin{aligned}
\tilde{q}_{A}(x, y)= & -\frac{1}{6} \frac{(1-x+\sqrt{3} y)^{2}(2 x+1)}{\left[\frac{1}{\sqrt{3}}(1-x+\sqrt{3} y)+\frac{1}{\sqrt{3}}(2 x+1)\right]^{2}} N_{2}[f]\left(\boldsymbol{p}_{B_{1}}(x, y)\right) \\
& -\frac{1}{6} \frac{(1-x+\sqrt{3} y)(2 x+1)^{2}}{\left[\frac{1}{\sqrt{3}}(1-x+\sqrt{3} y)+\frac{1}{\sqrt{3}}(2 x+1)\right]^{2}} N_{3}[f]\left(\boldsymbol{p}_{C_{1}}(x, y)\right), \\
\tilde{q}_{B}(x, y)= & -\frac{1}{6} \frac{(1-x+\sqrt{3} y)^{2}(1-x-\sqrt{3} y)}{\left[\frac{1}{\sqrt{3}}(1-x+\sqrt{3} y)+\frac{1}{\sqrt{3}}(1-x-\sqrt{3} y)\right]^{2}} N_{1}[f]\left(\boldsymbol{p}_{A_{2}}(x, y)\right) \\
& -\frac{1}{6} \frac{(1-x+\sqrt{3} y)(1-x-\sqrt{3} y)^{2}}{\left[\frac{1}{\sqrt{3}}(1-x+\sqrt{3} y)+\frac{1}{\sqrt{3}}(1-x-\sqrt{3} y)\right]^{2}} N_{3}[f]\left(\boldsymbol{p}_{C_{2}}(x, y)\right),
\end{aligned}
$$




$$
\begin{aligned}
\tilde{q}_{C}(x, y)= & -\frac{1}{6} \frac{(1-x-\sqrt{3} y)(2 x+1)^{2}}{\left[\frac{1}{\sqrt{3}}(1-x-\sqrt{3} y)+\frac{1}{\sqrt{3}}(2 x+1)\right]^{2}} N_{1}[f]\left(\boldsymbol{p}_{A_{3}}(x, y)\right) \\
& -\frac{1}{6} \frac{(1-x-\sqrt{3} y)^{2}(2 x+1)}{\left[\frac{1}{\sqrt{3}}(1-x-\sqrt{3} y)+\frac{1}{\sqrt{3}}(2 x+1)\right]^{2}} N_{2}[f]\left(\boldsymbol{p}_{B_{3}}(x, y)\right),
\end{aligned}
$$

The function $\tilde{q}_{A}$ interpolates the normal derivative of $f$ along edges 2 and 3, i.e., $N_{2}\left[\tilde{q}_{A}\right]=$ $N_{2}[f]$ and $N_{3}\left[\tilde{q}_{A}\right]=N_{3}[f]$, and has vanishing Dirichlet values along these edges. It reduces to a cubic polynomial along the first edge. The other functions $\tilde{q}_{B}$ and $\tilde{q}_{C}$ enjoy similar properties: they interpolate the normal of $f$ along two edges and reduce to cubic polynomials along the remaining edge.

However, the singularity of the function $\tilde{q}_{A}$ along the straight line $2+x+\sqrt{3} y=0$, which is parallel to the first edge and passes through $P_{23}$, is now removable only at the vertex $P_{23}$ itself. An interpolant with singularities near the triangle is certainly less than desirable. The situation can be remedied by enforcing additional conditions on $f$. In particular, further analysis of the singularities of $\tilde{q}_{A}$ shows that they are removable when $N_{2} f\left(P_{23}\right)=N_{3} f\left(P_{23}\right)=$ $T_{2} N_{2} f\left(P_{23}\right)+T_{3} N_{3} f\left(P_{23}\right)=0$. Taking into account the other functions $\tilde{q}_{B}$ and $\tilde{q}_{C}$ as well, all singularities are removable everywhere if

$$
\begin{aligned}
& N_{1} f\left(P_{12}\right)=N_{2} f\left(P_{23}\right)=N_{3} f\left(P_{31}\right)=0, \\
& N_{2} f\left(P_{12}\right)=N_{3} f\left(P_{23}\right)=N_{1} f\left(P_{31}\right)=0, \\
& T_{1} N_{1} f\left(P_{12}\right)+T_{2} N_{2} f\left(P_{12}\right)=0, \\
& T_{2} N_{2} f\left(P_{23}\right)+T_{3} N_{3} f\left(P_{23}\right)=0, \\
& T_{3} N_{3} f\left(P_{31}\right)+T_{1} N_{1} f\left(P_{31}\right)=0 .
\end{aligned}
$$

We may perform polynomial subtraction at the vertices once more to ensure that the conditions (4.7) hold. For example, we apply the interpolation procedure to $\tilde{f}=f-p$ rather than $f$, where $p(x, y)$ is a polynomial with 9 degrees of freedom of the form

$p(x, y)=a_{1} x^{2} y+a_{2} x^{3} y+a_{3} x^{2} y^{2}+a_{4} x^{5}+a_{5} x^{4} y+a_{6} x^{3} y^{2}+a_{7} x^{2} y^{3}+a_{8} x y^{4}+a_{9} y^{5}$.

The coefficients can be determined such that the 9 conditions (4.7) hold for $\tilde{f}$.

Finally, adding the functions $\tilde{q}_{A}, \tilde{q}_{B}$ and $\tilde{q}_{C}$ results in a function that interpolates twice the normal derivatives of $f$ along the edges plus an additional cubic term. We let

$$
\begin{aligned}
\tilde{q}(x, y)= & \frac{1}{2}\left[\tilde{q}_{A}(x, y)+\tilde{q}_{B}(x, y)+\tilde{q}_{C}(x, y)\right] \\
& -\frac{\sqrt{3}}{45}\left(-y^{2}+8 x y^{2}+8 x^{2} y^{2}+2 y^{4}\right) T_{1} N_{1} f\left(P_{31}\right) \\
- & \frac{1}{360}\left(-12 x y+4 \sqrt{3} y^{2}-20 \sqrt{3} x^{3}+24 x^{2} y+28 \sqrt{3} x y^{2}+24 y^{3}+15 \sqrt{3} x^{4}\right. \\
& \left.\quad-12 x^{3} y-2 \sqrt{3} x^{2} y^{2}+60 x y^{3}+7 \sqrt{3} y^{4}\right) T_{2} N_{2} f\left(P_{12}\right) \\
- & \frac{1}{360}\left(12 x y+4 \sqrt{3} y^{2}-20 \sqrt{3} x^{3}-24 x^{2} y+28 \sqrt{3} x y^{2}-24 y^{3}+15 \sqrt{3} x^{4}\right. \\
& \left.+12 x^{3} y-2 \sqrt{3} x^{2} y^{2}-60 x y^{3}+7 \sqrt{3} y^{4}\right) T_{3} N_{3} f\left(P_{31}\right)
\end{aligned}
$$



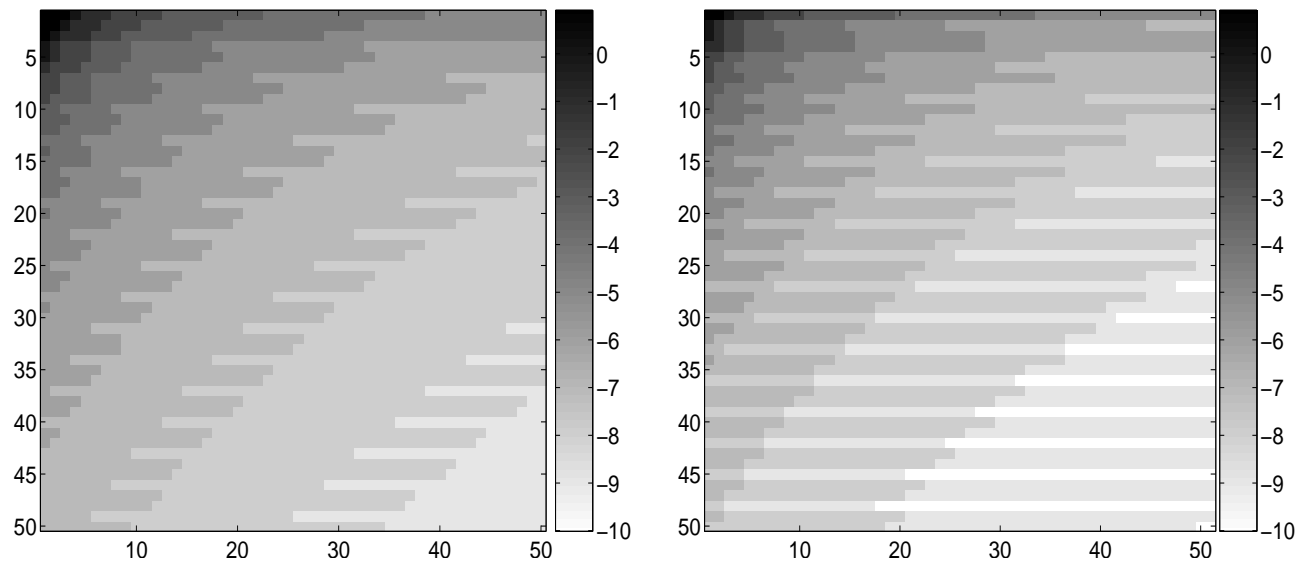

Figure 4.4: Plot of the base-10 logarithm of the size of the coefficients $c_{m, n}$ (left) and $s_{m, n}$ (right) for the function $f(x, y)-p(x, y)-\tilde{q}(x, y)=\mathrm{e}^{\pi(x-y)}-p(x, y)-\tilde{q}(x, y)$ in the $(m, n)$ plane, where $p$ interpolates $f$ at the vertices so that conditions (4.7) hold, and $\tilde{q}$ interpolates the normal derivative of $\tilde{f}=f-p$ along the boundary.

Straightforward, but exceedingly tedious, algebraic computations confirm that $N_{j}[\tilde{q}]=N_{j}[f]$, $j=1,2,3$, and that $\tilde{q}(x, y)$ is a smooth function everywhere in $x$ and $y$, assuming that the conditions (4.7) hold. Recall that these conditions can always be enforced by using polynomial subtraction at the vertices.

The results for the function $f(x, y)=\mathrm{e}^{\pi(x-y)}$ are shown in Figure 4.4. This figure should be compared to Figures 4.2 and 4.3. Note that the decay rate for small $m$ and increasing $n$, visible in the first few rows of the figures, is drastically improved. The decay rate for increasing $m$ is also improved compared to polynomial subtraction at the vertices only.

\section{The computation of coefficients}

An outstanding issue is the computation of modified Fourier coefficients, as given by the integrals (2.5). For large $m$ and/or $n$, these integrals become exceedingly oscillatory. Fortunately, and perhaps surprisingly, the evaluation of such integrals is a fairly cheap operation. The existence of the asymptotic expansions constructed in $\S 3$ already indicates that the computational cost of evaluating such integrals should be fixed with increasing oscillation. Several numerical methods have been designed for univariate oscillatory integrals that exhibit precisely this property (Huybrechs \& Olver 2009). Like for asymptotic expansions, accuracy rapidly improves with increasing oscillation. These methods can in principle be all extended to the current setting, though in previous papers we restricted our attention to the Filon-type methods of (Iserles \& Nørsett 2005). They take the form of a classical cubature rule using derivatives. In the context of modified Fourier on the equilateral triangle, the derivatives to use in Filon-type cubature are exactly those that appear in the asymptotic expansions of $\S 3$.

With efficient methods for oscillatory integrals available, it is the evaluation of nonoscillatory integrals that presents the next computational hurdle. Many numerical integration 
schemes for a triangular domain have been described in the literature, we refer the reader to (Lyness \& Cools 1993) for a survey. It is wasteful however to have separate schemes, each with their own set of cubature points, for non-oscillatory and oscillatory regimes. For the sake of efficiency, our aim is to reuse information as much as possible and, hence, to match the integration schemes. Since derivatives are required in Filon-type methods for highly oscillatory integrals, we will reuse that information in the computation of non-oscillatory integrals. A first step in this direction led to exotic quadrature in (Iserles \& Nørsett 2008). In this paper we extend the concept of exotic quadrature to the equilateral triangle and we construct new cubature rules using derivatives for the equilateral triangle.

\subsection{Filon-type cubature}

The idea underlying most cubature schemes is to interpolate the integrand at cubature points and to integrate that interpolant exactly (Davis \& Rabinowitz 1984). In Filon-type cubature, in addition to this, one interpolates all data on which the first few terms of the asymptotic expansion of the integral depend. Thus, with expressions (4.1) and (4.2) in mind, consider a polynomial $p$ that satisfies

$$
\begin{aligned}
& \left(T_{1}+T_{2}\right) p\left(P_{12}\right)=\left(T_{1}+T_{2}\right) f\left(P_{12}\right), \\
& \left(T_{2}+T_{3}\right) p\left(P_{23}\right)=\left(T_{2}+T_{3}\right) f\left(P_{23}\right), \\
& \left(T_{3}+T_{1}\right) p\left(P_{31}\right)=\left(T_{3}+T_{1}\right) f\left(P_{31}\right),
\end{aligned}
$$

in addition to

$$
\partial_{\boldsymbol{x}}^{\boldsymbol{j}_{k}} p\left(\boldsymbol{x}_{k}\right)=\partial_{\boldsymbol{x}^{k}}^{\boldsymbol{j}_{k}} f\left(\boldsymbol{x}_{k}\right), \quad k=1, \ldots, \nu,
$$

for a set of cubature points $\boldsymbol{x}_{k}$ and corresponding partial derivatives of order $\boldsymbol{j}_{k}$. We define the Filon-type cubature rule $Q_{F}^{[m, n]}[f]$ by

$$
Q_{F}^{[m, n]}[f]=\int_{T} p(\boldsymbol{x}) \psi_{m, n}(\boldsymbol{x}) \mathrm{d} V .
$$

It follows from the asymptotic expansions that

$$
\left\langle f, \psi_{m, n}\right\rangle-Q_{F}^{[m, n]}[f]=\mathcal{O}\left(m^{-4}\right) .
$$

Thus, the accuracy of Filon-type quadrature improves with increasing $m$, but the computational cost remains fixed. Note that the moments in the right hand side of (5.2) may be computed from their asymptotic expansions, because the asymptotic expansion for polynomial $f$ has only finitely many terms.

Expressions (5.1) correspond exactly to the interpolation conditions (4.4) for convergence acceleration that appeared in $\S 4$. Higher order convergence of Filon-type cubature can be obtained by satisfying the more general conditions (4.5). The difference compared to convergence acceleration is the freedom we have introduced to use additional cubature points. This freedom can be used to improve accuracy. However, this is subject to the solvability of the underlying interpolation problem, which cannot be taken for granted.

In particular, the construction of Filon-type cubature is plagued by two issues. Firstly, the use of non-consecutive derivatives leads to a Birkhoff-Hermite interpolation problem (Lorenz, Jetter \& Riemenschneider 1983). The interpolation problem is not necessarily solvable by any 
polynomial with a number of degrees of freedom that matches the number of interpolation conditions, even in one dimension. Secondly, a multivariate interpolating polynomial need not exist for a particular configuration of points, even disregarding the use of derivatives. Both issues were dealt with for multivariate domains with tensor-product structure in (Iserles \& Nørsett 2007). The techniques of $\S 4.2$ of that paper (i.e., reinterpreting Filon-type cubature as a correction to the truncated asymptotic expansion) are equally applicable, at least in principle, to the case of the equilateral triangle. In the current paper, we leave these issues outstanding and focus on a description of new exotic cubature rules using the same kind of information.

\subsection{Exotic cubature}

\subsubsection{General considerations}

The construction of cubature rules for multivariate domains is very different from that of quadrature rules in one dimension. For an overview of relevant techniques, we refer the reader to (Cools 1997). Cubature rules on triangular domains are surveyed in (Lyness \& Cools 1993). Several cubature rules of Gauss-type were obtained in (Lyness \& Jespersen 1975), many others can be found in the encyclopedic list (Cools 2003).

A cubature rule on a two-dimensional region $\Omega$,

$$
Q[f]:=\sum_{j=1}^{n} w_{j} f\left(x_{j}, y_{j}\right)
$$

is said to have degree $d$ if it is exact for the $\frac{1}{2}(d+1)(d+2)$ polynomials of total degree less than $d$, i.e.,

$$
Q\left[x^{i} y^{j}\right]=\int_{\Omega} x^{i} y^{j} \mathrm{~d} x \mathrm{~d} y, \quad 0 \leq i, j \leq d, \quad i+j \leq d .
$$

A classical problem is to construct a rule of degree $d$ that requires the fewest function evaluations. Rules of specific interest are those with all weights positive and with all cubature points inside the domain of integration. Unlike in the one-dimensional case, it is unknown in general what the minimal number of cubature points is for a given degree $d$, though for any two-dimensional region the value $\frac{1}{2}\left(\frac{d}{2}+1\right)\left(\frac{d}{2}+2\right)$ is a lower bound (Stroud 1971).

Cubature rules may be computed by solving the nonlinear set of algebraic equations (5.5), with the weights and cubature points as degrees of freedom. The dimensionality of this nonlinear system may be reduced by exploiting the symmetries of the integration domain.

\subsubsection{Symmetries of the triangle}

Recall that the triangle $T$ is kept invariant under the action of the dihedral group $D_{3}$, composed of six elements: identity, rotations $R$ and $R^{-1}$ by $\frac{2}{3} \pi$ and $-\frac{2}{3} \pi$ radians respectively, and reflections $Q_{i, i+1}, i=1,2,3$, with respect to axes of symmetry passing through the vertices $P_{i, i+1}$ respectively. For the record, the exact symmetries are

$$
\begin{aligned}
R(x, y) & =\left(-\frac{1}{2} x+\frac{\sqrt{3}}{2} y,-\frac{\sqrt{3}}{2} x-\frac{1}{2} y\right), \\
R^{-1}(x, y) & =\left(-\frac{1}{2} x-\frac{\sqrt{3}}{2} y, \frac{\sqrt{3}}{2} x-\frac{1}{2} y\right), \\
Q_{12}(x, y) & =\left(-\frac{1}{2} x-\frac{\sqrt{3}}{2} y,-\frac{\sqrt{3}}{2} x+\frac{1}{2} y\right),
\end{aligned}
$$




$$
\begin{aligned}
& Q_{23}(x, y)=\left(-\frac{1}{2} x+\frac{\sqrt{3}}{2} y, \frac{\sqrt{3}}{2} x+\frac{1}{2} y\right), \\
& Q_{31}(x, y)=(x,-y) .
\end{aligned}
$$

A cubature rule with the same symmetries can be written as

$$
Q[f]=\sum_{j=1}^{n} a_{j} \sum_{i=1}^{6} f\left(S_{i}\left(x_{j}, y_{j}\right)\right),
$$

where $S_{1}=I, S_{2}=R, S_{3}=R^{-1}, S_{4}=Q_{12}, S_{5}=Q_{23}, S_{6}=Q_{31}$ are all the dihedral symmetries. Each point $(x, y)$ leads to $\imath(x, y)$ function evaluations, where $\imath$ is 6 divided by the dimension of the isotropy group at $(x, y)$ : thus, $\imath(0,0)=1, \imath(x, y)=3$ for $(x, y)$ on the three axes of reflectional symmetry and $\imath(x, y)=6$ otherwise.

The symmetries of the equilateral triangle were first exploited this way in (Lyness \& Jespersen 1975). The $\frac{1}{2}(d+1)(d+2)$ conditions (5.5) reduce to just

$$
\left\lfloor\frac{1}{12}\left(d^{2}+6 d+12\right)\right\rfloor
$$

nontrivial equations. The gain is approximately a factor of 6 .

\subsubsection{Cubature rules}

Mildly generalizing the above expressions in order to use derivatives, we are interested in cubature rules of the form

$$
Q[f]=\sum_{j=1}^{n} a_{j} \rho^{\left[\boldsymbol{m}_{j}\right]}\left(\boldsymbol{c}_{j}\right)
$$

with

$$
\rho^{[\boldsymbol{m}]}(\boldsymbol{c})=\frac{\sqrt{3}}{6} \sum_{i=1}^{6} N^{m_{1}} T^{m_{2}} f\left(S_{i} \boldsymbol{c}\right) .
$$

We impose the rule that derivatives may be used only at the vertices and, more generally, also along the axes of reflectional symmetry. With this restriction, we adopt the convention that $N$ and $T$ denote the normal and tangential derivatives associated with the edge opposite the vertex $c$, or with the edge orthogonal to the axes of reflectional symmetry on which $c$ lies. Note that the factor $\sqrt{3}$ in (5.7) serves to simplify the expressions for the coefficients $a_{j}$.

Table 1: Order and number of function evaluations of the cubature rules using derivatives.

\begin{tabular}{c|cc||c|cc|} 
rule & order & function evaluations & rule & order & function evaluations \\
\hline$Q_{1}$ & 5 & 7 & $Q_{6}$ & 9 & 34 \\
$Q_{2}$ & 6 & 16 & $Q_{7}$ & 11 & 57 \\
$Q_{3}$ & 7 & 19 & $Q_{8}$ & 12 & 64 \\
$Q_{4}$ & 7 & 22 & $Q_{9}$ & 12 & 61 \\
$Q_{5}$ & 8 & 28 & & & \\
\hline
\end{tabular}




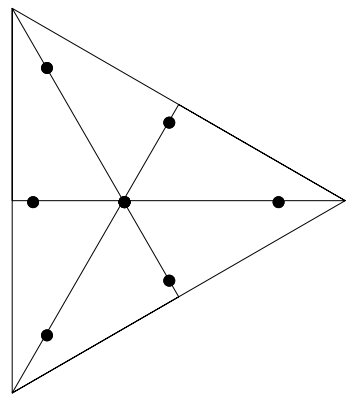

(a) $Q_{1}$, order 5

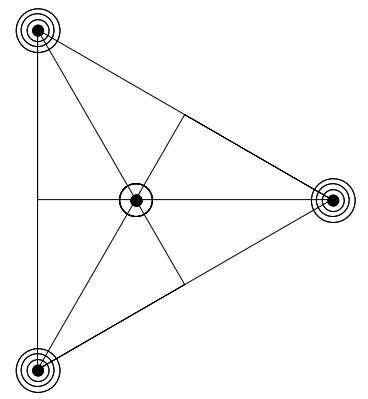

(d) $Q_{4}$, order 7

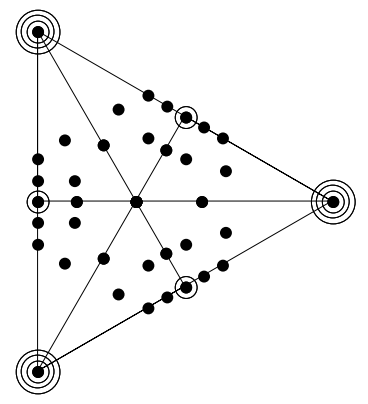

(g) $Q_{7}$, order 11

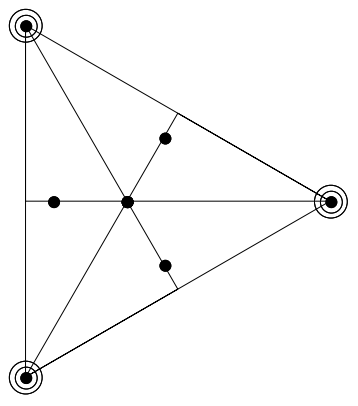

(b) $Q_{2}$, order 6

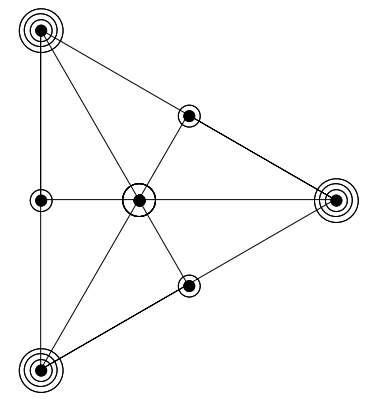

(e) $Q_{5}$, order 8

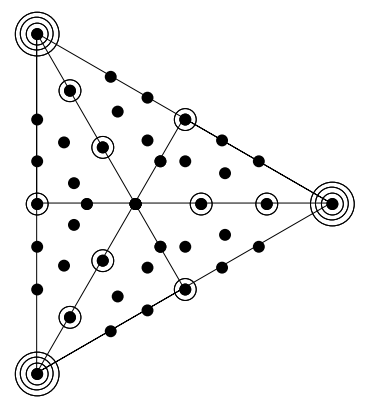

(h) $Q_{8}$, order 12

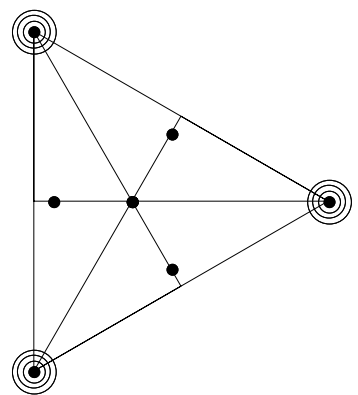

(c) $Q_{3}$, order 7

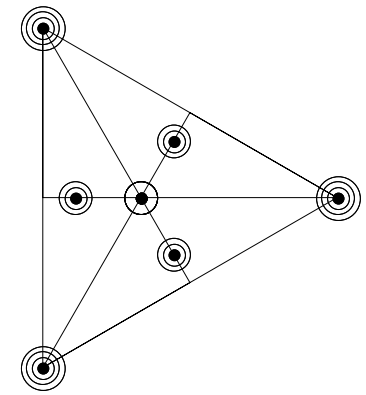

(f) $Q_{6}$, order 9

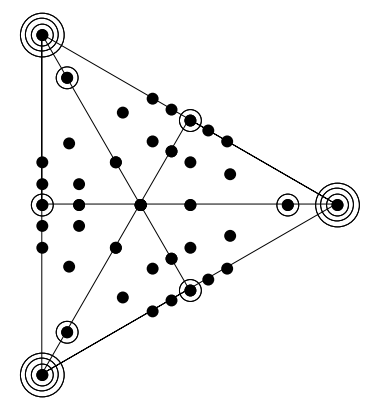

(i) $Q_{9}$, order 12

Figure 5.1: Illustration of the location of the cubature points.

It is unknown in general what is the minimal number of points such that the conditions (5.5) hold up to a certain degree. The cubature rules in this paper correspond only to local minima. They were found by iteratively solving small and local optimization problems. Starting from a given cubature rule, cubature points were removed by varying other cubature points in a small neighbourhood of their location, restricted to certain lines to further simplify the computations. It is likely that this fairly ad hoc and manual approach can be considerably improved. Yet, cubature rules using derivatives were found up to order 12 .

Cubature rules of orders between 5 and 12 are depicted graphically in Figure 5.1. The 
cubature points are indicated by dots. Derivatives of increasing order are indicated by circles with increasing radius. These rules all have the form (5.6). The precise values of the parameters $a_{j}, \boldsymbol{m}_{j}$ and $\boldsymbol{c}_{j}$ are tabulated in Appendix A. We note that the first cubature rule $Q_{1}$ is known (Abramowitz \& Stegun 1965, p.893). Table 1 summarizes the order and the total number of function evaluations of the cubature rules depicted in Figure 5.1. In this table, evaluations of derivatives are counted as function evaluations.

\subsection{Copy rules}

Convergence in numerical integration is not obtained usually by increasing the order of the rules, but by subdividing the integration region. The equilateral triangle can be divided into four smaller equilateral triangles, termed $A, B, C$ and $D$ in Figure 5.2. A cubature rule on the larger triangle can, when appropriately rescaled, be used on each of these triangles separately. This leads to so-called copy rules.

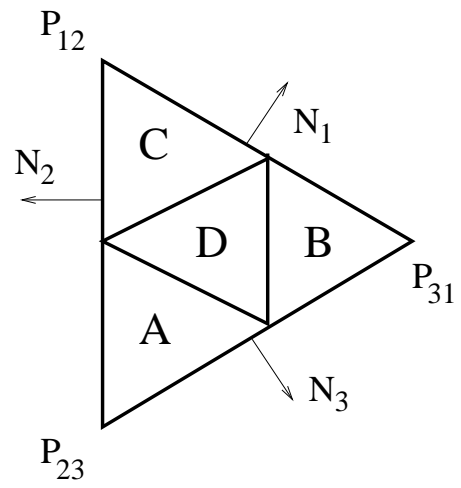

Figure 5.2: Subdivision of the equilateral triangle into four smaller equilateral triangles.

The scaling of a cubature rule to a smaller domain usually requires only multiplying the weights with a factor proportional to the size of that domain. However, when using (directional) derivatives, rescaling cubature rules is slightly more involved. To be precise, each function evaluation in the exotic cubature rules constructed above takes the form

$$
w N_{i}^{m_{1}} T_{i}^{m_{2}} f(\boldsymbol{c}),
$$

for some value of $w$ and with $i=1,2$ or 3 . Consider the unique affine mappings $P_{A}, P_{B}$, $P_{C}$ and $P_{D}$ that map the equilateral triangle to each of the smaller triangles, such that $P_{A}$, $P_{B}$ and $P_{C}$ preserve the normal directions of the three edges and $P_{D}$ mirrors these directions with respect to the vertical axis. Then it is straightforward to demonstrate that the function evaluation (5.8) results in the function evaluation

$$
\frac{1}{4}\left(\frac{1}{2}\right)^{m_{1}+m_{2}} N_{i}^{m_{1}} T_{i}^{m_{2}} f\left(P_{A} \boldsymbol{c}\right),
$$

when the cubature rule is rescaled to triangle $A$. Similar expressions hold for triangles $B$ and $C$. The factor $1 / 4$ is due to the smaller size of triangle $A$. The additional powers of $1 / 2$ are due to the derivatives taken. 
The case of triangle $D$ is different, because $P_{D}$ maps $N_{1}$ to $-N_{3}, N_{2}$ to $-N_{2}$ and $N_{3}$ to $-N_{1}$. In this case, the above function evaluation becomes

$$
\frac{1}{4}\left(-\frac{1}{2}\right)^{m_{1}+m_{2}} N_{j}^{m_{1}} T_{j}^{m_{2}} f\left(P_{D} \boldsymbol{c}\right),
$$

with $j=3,2,1$ for $i=1,2,3$ respectively. Note that both evaluations (5.9) and (5.10) have the same general form as (5.8).

\subsection{Numerical results}

First, we illustrate the use of exotic cubature for the evaluation of the integral of $f(x, y)=$ $J_{0}\left(x^{2}+x y-\cos (x+y)\right)$ over the equilateral triangle, where $J_{0}(z)$ is the Bessel function of the first kind and order 0 . Results are shown in Table 2 for the cubature rules constructed in this paper. The table illustrates the effect of increasing the number of levels in copy rules. Level $l=0$ corresponds to the cubature rule itself. Level $l=1$ means that the triangle is subdivided into four smaller triangles. Level $l=2$ means that each of the smaller triangles is again subdivided, and so on.

Table 2: Absolute error in the approximation of $\int_{T} J_{0}\left(x^{2}+x y-\cos (x+y)\right) \mathrm{d} x \mathrm{~d} y$ by cubature rules using derivatives. Copy rules are constructed of $l=0,1,2$ or 3 levels deep.

\begin{tabular}{c|cccc||c|cccc}
\hline rule & $l=0$ & $l=1$ & $l=2$ & $l=3$ & rule & $l=0$ & $l=1$ & $l=2$ & $l=3$ \\
\hline$Q_{1}$ & $3.5_{-04}$ & $1.6_{-05}$ & $2.8_{-07}$ & $4.4_{-09}$ & $Q_{6}$ & $4.9_{-05}$ & $4^{-08}$ & $3.3_{-11}$ & $2.6_{-14}$ \\
$Q_{2}$ & $1.7_{-03}$ & $7.9_{-07}$ & $4.8_{-10}$ & $4.2_{-12}$ & $Q_{7}$ & $5.8_{-06}$ & $1^{-09}$ & $4.9_{-13}$ & $8.2_{-17}$ \\
$Q_{3}$ & $5.4_{-04}$ & $4.7_{-07}$ & $5.4_{-10}$ & $1.2_{-12}$ & $Q_{8}$ & $7.1_{-07}$ & $6.2_{-11}$ & $2.7_{-16}$ & $1.2_{-20}$ \\
$Q_{4}$ & $3.2_{-03}$ & $2.9_{-06}$ & $8.0_{-10}$ & $1.1_{-11}$ & $Q_{9}$ & $3.8_{-07}$ & $2.0_{-11}$ & $4.7_{-16}$ & $2.7_{-20}$ \\
$Q_{5}$ & $1.7_{-04}$ & $1.6_{-07}$ & $1.0_{-10}$ & $9.0_{-14}$ & & & & & \\
\hline
\end{tabular}

Next, we illustrate the convergence rate of the modified Fourier series for the same function $f(x, y)$. In this experiment we truncated the series (2.4) at $m=N$ and $n=N$, where $N$ is a constant. Pointwise convergence is illustrated in Fig. 5.3 for increasing $N$ at three points in the triangle: a point in the interior, a point on one of the edges and a vertex. The figure shows the modified Fourier series of $\tilde{f}=f-p-\tilde{q}$, with $p$ and $\tilde{q}$ constructed so that the normal derivatives vanish along the boundary of the triangle. The convergence at the interior point is $\mathcal{O}\left(N^{-4}\right)$, which is consistent with the size of the leading order term of the asymptotic expansion after polynomial subtraction. Pointwise convergence along the boundary is slower: it is $\mathcal{O}\left(N^{-3}\right)$ for both points on the boundary. This is expected and is similar to modified Fourier series on the interval and on multivariate domains with tensor-product structure.

Finally, we illustrate the accuracy of the asymptotic expansions constructed in this paper for the function $f(x, y)$. Let us denote by $\mathcal{A}_{s}^{[m, n]}$ the expansion (3.4) for $c_{m, n}$ truncated after $s$ terms in the outer summation (i.e., $k=0, \ldots, s-1$ ), and similarly by $\mathcal{E}_{s}^{[m, n]}$ the truncated expansion for the edge coefficients based on (3.8). The results are shown in Table 3. It is clearly visible in this table that the accuracy of the expansions rapidly improves with increasing $m$ and/or $n$. It is to be expected that Filon-type cubature rules would result in similar improvements, but with significantly smaller values of the errors. 


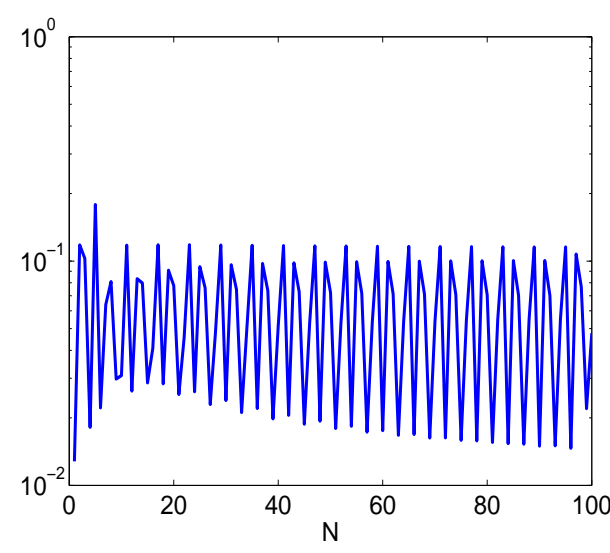

(a) Absolute error at $\left(\frac{1}{2}, 0\right)$, scaled by $N^{4}$

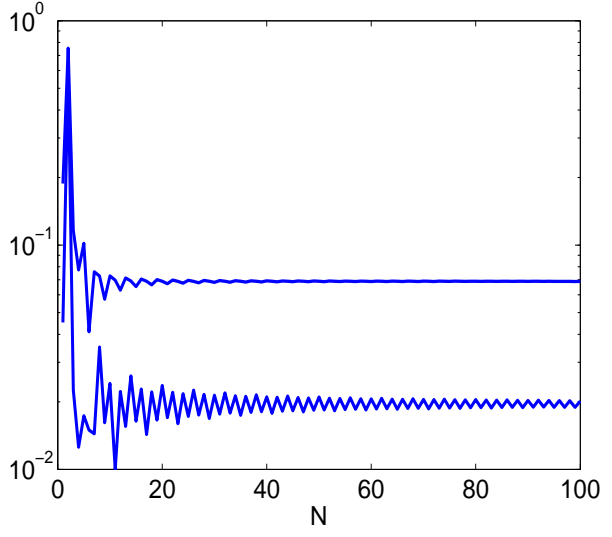

(b) Absolute error at vertex $(1,0)$ (top curve) and at $\left(-\frac{1}{2}, 0\right)$ (bottom curve), scaled by $N^{3}$

Figure 5.3: Pointwise convergence of the modified Fourier series of $J_{0}\left(x^{2}+x y-\cos (x+y)\right)$ with polynomial subtraction. The doubly-infinite series (2.4) is truncated at $m=N$ and $n=N$. Convergence is shown for increasing $N$ in three points: a point in the interior (left panel) and two points along the edges (right panel).

The modified Fourier series edge coefficients in this example were computed by using copy rules with a large number of levels. This of course does not lead to an $\mathcal{O}\left(N^{2}\right)$ scheme to compute the $N^{2}$ coefficients. These brute-force computations were performed in order to compare with results obtained by using the asymptotic expansion. The cost of evaluating the asymptotic expansion remains fixed for each coefficient, regardless of the value of $m$ and $n$.

Table 3: Absolute error of the asymptotic expansions for the modified Fourier series coefficients of $f(x, y)=J_{0}\left(x^{2}+x y-\cos (x+y)\right)$.

\begin{tabular}{c|lll||l|lll|}
{$[m, n]$} & $\mathcal{A}_{1}^{[m, n]}$ & $\mathcal{A}_{2}^{[m, n]}$ & $\mathcal{A}_{3}^{[m, n]}$ & {$[m, n]$} & $\mathcal{E}_{1}^{[m, n]}$ & $\mathcal{E}_{2}^{[m, n]}$ & $\mathcal{E}_{3}^{[m, n]}$ \\
\hline \hline$[5,0]$ & $8.7_{-04}$ & $4.2_{-04}$ & $2.2_{-04}$ & {$[0,5]$} & $2.6_{-04}$ & $8.1_{-06}$ & $5.1_{-08}$ \\
{$[10,0]$} & $2.3_{-05}$ & $3.9_{-06}$ & $6.4_{-07}$ & {$[0,10]$} & $1.6_{-05}$ & $1.3_{-07}$ & $2.6_{-10}$ \\
{$[10,10]$} & $1.1_{-06}$ & $1.5_{-07}$ & $3.0_{-08}$ & {$[0,20]$} & $1.0_{-06}$ & $2.0_{-09}$ & $1.4_{-12}$ \\
{$[100,100]$} & $8.6_{-12}$ & $1.0_{-14}$ & $2.7_{-17}$ & {$[0,50]$} & $2.6_{-08}$ & $8.0_{-12}$ & $1.7_{-15}$ \\
\hline
\end{tabular}

\section{Conclusions}

The main result of this paper is the asymptotic expansion of coefficients in Laplace-Neumann series (or modified Fourier series) on the equilateral triangle. This expansion conveys the information necessary to accelerate the convergence of such series, to efficiently compute the 
coefficients and to predict and explain the shape of the hyperbolic cross in this setting. First steps were already taken in these directions using the techniques of polynomial subtraction, Filon-type cubature and exotic cubature. Taken together these steps have not currently led in this paper to a complete, fast and competitive implementation of a Laplace-Neumann approximation scheme. In particular, such a scheme would entail a constructive approach for high-order convergence acceleration and fully matched integration schemes for slowly and highly oscillatory integrals. Yet, based on the knowledge of the asymptotic expansion and on the foundations laid in this paper, these missing elements appear to be within reach and they are the subject of further research.

\section{Acknowledgements}

We wish to acknowledge insightful discussions with David Levin (Tel Aviv) on ComputerAided Geometric Design techniques. Section 4.3 is a direct outcome of these discussions.

\section{References}

Abramowitz, M. \& Stegun, I. A. (1965), Handbook of Mathematical Functions with Formulas, Graphs, and Mathematical Tables, Dover Publications, New York.

Boyd, J. P. (2001), Chebyshev and Fourier Spectral Methods, Courier Dover Publications, Mineola, NY.

Cools, R. (1997), 'Constructing cubature formulae: The science behind the art', Acta Numer. 6, $1-54$

Cools, R. (2003), 'An encyclopedia of cubature formulas', Journal of Complexity 19, 445453.

Daubechies, I. (1992), Ten Lectures on Wavelets, SIAM, Philadelphia.

Davis, P. J. \& Rabinowitz, P. (1984), Methods of Numerical Integration, 2nd edn, Academic Press, Orlando, FL.

Farin, G. (1997), Curves and Surfaces for CAGD: A Practical Guide, 4th edn, Academic Press, San Diego.

Huybrechs, D. \& Olver, S. (2009), Highly oscillatory quadrature, in B. Engquist, A. Fokas, E. Hairer \& A. Iserles, eds, 'Highly Oscillatory Problems: Computation, Theory and Application', Cambridge Univ. Press, Cambridge, pp. 25-50.

Huybrechs, D. \& Vandewalle, S. (2006), 'On the evaluation of highly oscillatory integrals by analytic continuation', SIAM J. Num. Anal. 44, 1026-1048.

Huybrechs, D., Iserles, A. \& Nørsett, S. P. (2007), From high oscillation to rapid approximation IV: Accelerating convergence, Technical Report NA2007/07, DAMTP, University of Cambridge. 
Iserles, A. \& Nørsett, S. P. (2005), 'Efficient quadrature of highly oscillatory integrals using derivatives', Proc. Royal Soc. A 461, 1383-1399.

Iserles, A. \& Nørsett, S. P. (2006), From high oscillation to rapid approximation II: Expansions in polyharmonic eigenfunctions, Technical Report NA2006/05, DAMTP, University of Cambridge.

Iserles, A. \& Nørsett, S. P. (2007), From high oscillation to rapid approximation III: Multivariate expansions, Technical Report NA2007/01, DAMTP, University of Cambridge. To appear in IMA J. Num. Anal.

Iserles, A. \& Nørsett, S. P. (2008), 'From high oscillation to rapid approximation I: Modified Fourier expansions', IMA J. Num. Anal. 28, 862-887.

Lamé, G. (1833), 'Mémoire sur la propagation de la chaleur dans les polyédres', J. de École Polytechnique 22, 194-251.

Lorenz, G. G., Jetter, K. \& Riemenschneider, S. D. (1983), Birkhoff Interpolation, AddisonWesley, London.

Lyness, J. N. \& Cools, R. (1993), A survey of numerical cubature over triangles, in W. Gautschi, ed., 'Mathematics of Computation 1943-1993: A Half-Century of Computational Mathematics', Vol. 48 of Proceedings of Symposia in Applied Mathematics, American Mathematical Society, Providence, RI, pp. 127-150.

Lyness, J. N. \& Jespersen, D. (1975), 'Moderate degree symmetric quadrature rules for the triangle', IMA Journal of Applied Mathematics 15(1), 19-32.

Nikolov, G. (1989), 'Existence and uniqueness of Hermite-Birkhoff Gaussian quadrature formulas', Calcolo 26, 41-59.

Olver, S. (2006), 'Moment-free numerical integration of highly oscillatory functions', IMA J. Num. Anal. 26, 213-227.

Olver, S. (2009), 'On the convergence rate of a modified Fourier series', Math. Comp. To appear.

Práger, M. (1998), 'Eigenvalues and eigenfunctions of the Laplace operator on an equilateral triangle', Applications of Mathematics 43, 311-320.

Stroud, A. H. (1971), Approximate Calculation of Multiple Integrals, Prentice-Hall, Englewood Cliffs, N.J.

Temlyakov, V. (1993), Approximation of Periodic Functions, Nova Sci., New York.

\section{Appendix A}

The values of the parameters $a_{j}, \boldsymbol{m}_{j}$ and $\boldsymbol{c}_{j}$ of the general expression (5.6) of a cubature rule are shown in the table below for the cubature rules depicted in Figure 5.1. The values are given either in exact analytic form or rounded to 20 significant digits. 


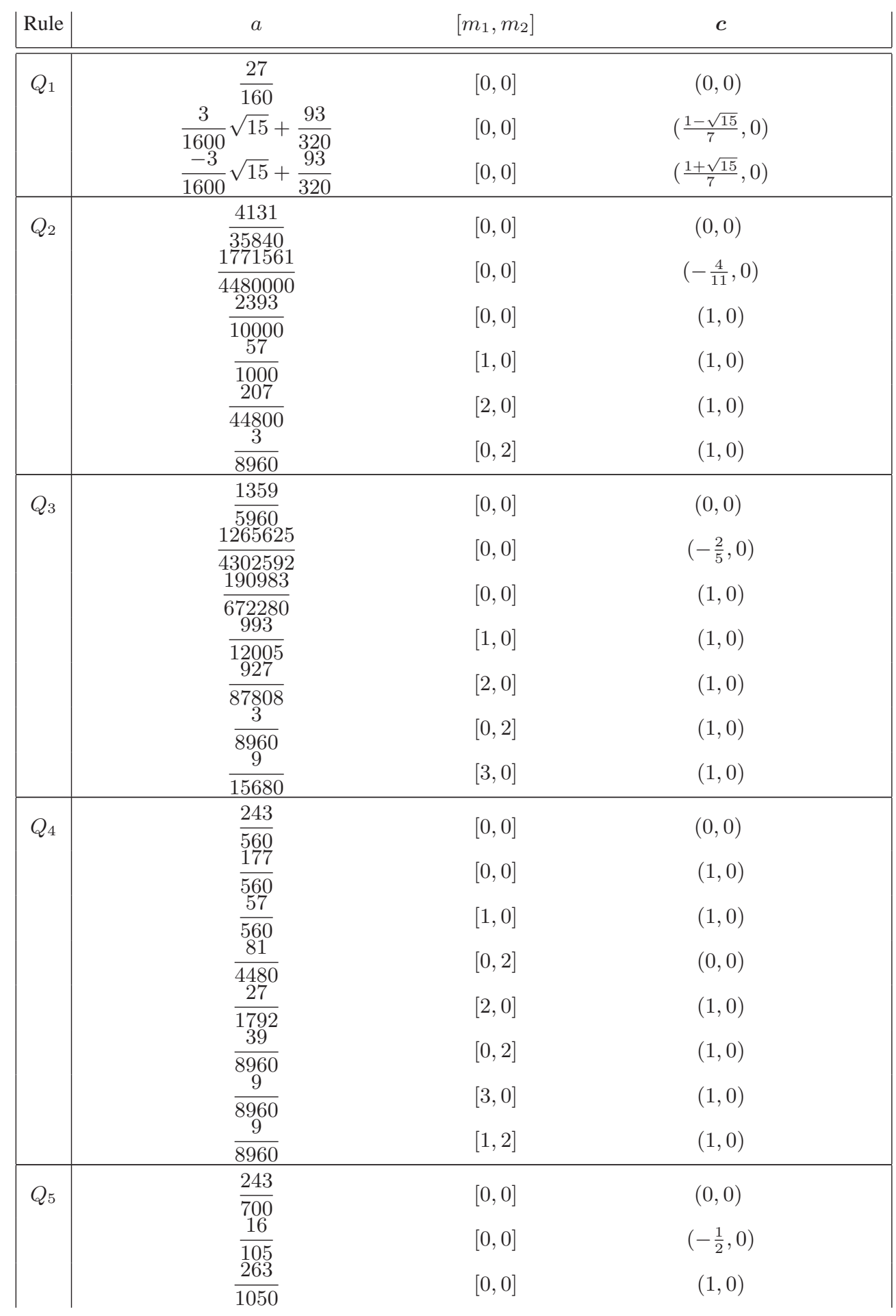




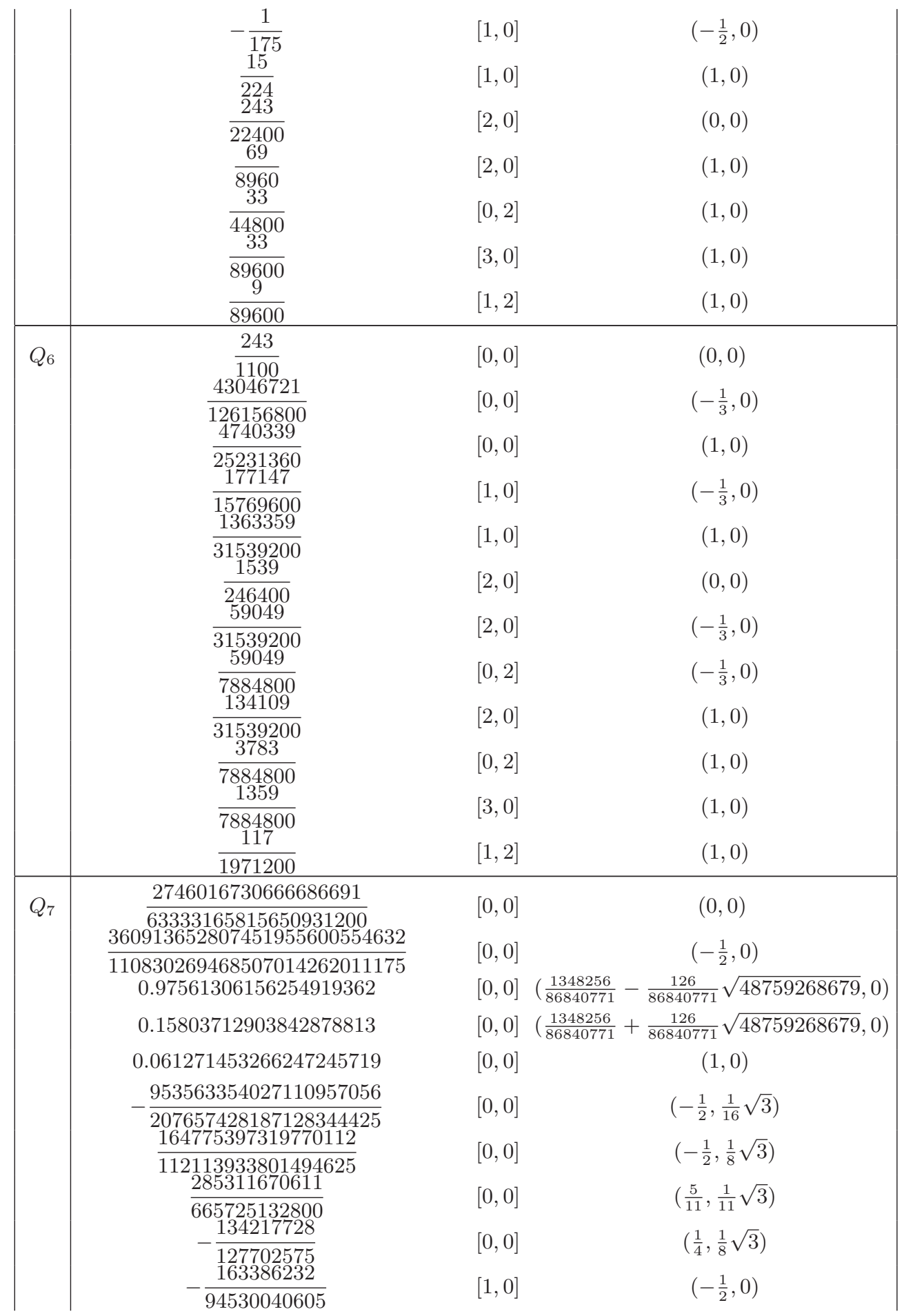




\begin{tabular}{|c|c|c|c|}
\hline & $\begin{array}{c}\frac{8319078131695973767147039560511}{1228985969075244255012191075221200} \\
\frac{73003115981986824353}{311081707624833223958880} \\
-\frac{71752743292487}{446462598605063040} \\
\quad-\frac{104380623}{5356544152960} \\
\end{array}$ & $\begin{array}{l}{[1,0]} \\
{[2,0]} \\
{[0,2]} \\
{[1,2]}\end{array}$ & $\begin{array}{l}(1,0) \\
(1,0) \\
(1,0) \\
(1,0)\end{array}$ \\
\hline$Q_{8}$ & $\begin{array}{c}\frac{8690571}{17937920} \\
\frac{229199798584}{1825082634375} \\
\frac{5280989184}{3709330625} \\
\frac{103545432999}{313913600000} \\
-\frac{1052102782197}{4581399961600} \\
-\frac{399141898759031}{490377888000000} \\
\frac{13019119616}{718326984375} \\
\frac{55181312}{1609052445} \\
\frac{285311670611}{665725132800} \\
-\frac{134217728}{127702575} \\
-\frac{10104148}{1931304375} \\
-\frac{150752097}{407680000} \\
-\frac{127816697763}{386741555200} \\
-\frac{566856277877}{4086482400000} \\
-\frac{1066688341}{121080960000} \\
\frac{2746573}{45405360000} \\
-\frac{1279519}{6054048000} \\
\frac{1601}{378378000}\end{array}$ & $\begin{array}{l}{[0,0]} \\
{[0,0]} \\
{[0,0]} \\
{[0,0]} \\
{[0,0]} \\
{[0,0]} \\
{[0,0]} \\
{[0,0]} \\
{[0,0]} \\
{[0,0]} \\
{[1,0]} \\
{[1,0]} \\
{[1,0]} \\
{[1,0]} \\
{[2,0]} \\
{[0,2]} \\
{[3,0]} \\
{[1,2]}\end{array}$ & $\begin{array}{c}(0,0) \\
\left(-\frac{1}{2}, 0\right) \\
\left(-\frac{1}{4}, 0\right) \\
\left(\frac{1}{3}, 0\right) \\
\left(\frac{2}{3}, 0\right) \\
(1,0) \\
\left(-\frac{1}{2}, \frac{1}{8} \sqrt{3}\right) \\
\left(-\frac{1}{2}, \frac{1}{4} \sqrt{3}\right) \\
\left(\frac{5}{11}, \frac{1}{11} \sqrt{3}\right) \\
\left(\frac{1}{4}, \frac{1}{8} \sqrt{3}\right) \\
\left(-\frac{1}{2}, 0\right) \\
\left(\frac{1}{3}, 0\right) \\
\left(\frac{2}{3}, 0\right) \\
(1,0) \\
(1,0) \\
(1,0) \\
(1,0)\end{array}$ \\
\hline$Q_{9}$ & $\begin{array}{c}-\frac{16035143725210437}{31243371390926356480} \\
\frac{3230803273641114501493}{6168424871724534168750} \\
0.94606756593949053915 \\
\frac{50422996864}{211843372125} \\
\frac{724750010853899359104}{19256701191020490071875} \\
0.068019898691773271037 \\
-\frac{231447197646848}{312930128556045}\end{array}$ & $\begin{array}{l}{[0,0]} \\
{[0,0]} \\
{[0,0]} \\
{[0,0]} \\
{[0,0]} \\
{[0,0]} \\
{[0,0]}\end{array}$ & $\begin{array}{c}(0,0) \\
\left(-\frac{1}{2}, 0\right) \\
\left(-\frac{2188564}{6966959}, 0\right) \\
\left(\frac{1}{4}, 0\right) \\
\left(\frac{3}{4}, 0\right) \\
(1,0) \\
\left(-\frac{1}{2}, \frac{1}{16} \sqrt{3}\right)\end{array}$ \\
\hline
\end{tabular}




\begin{tabular}{|ccc|}
$\frac{1503775424512}{5028288890625}$ & {$[0,0]$} & $\left(-\frac{1}{2}, \frac{1}{8} \sqrt{3}\right)$ \\
$\frac{285311670611}{665725132800}$ & {$[0,0]$} & $\left(\frac{5}{11}, \frac{1}{11} \sqrt{3}\right)$ \\
$-\frac{134217728}{127702575}$ & {$[0,0]$} & $\left(\frac{1}{4}, \frac{1}{8} \sqrt{3}\right)$ \\
$-\frac{103949931131}{136102093627500}$ & {$[1,0]$} & $\left(-\frac{1}{2}, 0\right)$ \\
$\frac{122010152256}{129870948081875}$ & {$[1,0]$} & $\left(\frac{3}{4}, 0\right)$ \\
0.0097348092854897992901 & {$[1,0]$} & $(1,0)$ \\
$\frac{561653255177898184373029}{949434177302358293669760000}$ & {$[2,0]$} & $(1,0)$ \\
$\frac{33780569353}{420499038960000}$ & {$[0,2]$} & $(1,0)$ \\
$\frac{156887373995423}{10863883238592384000}$ & {$[3,0]$} & $(1,0)$ \\
$\frac{319547}{55521566000}$ & {$[1,2]$} & $(1,0)$ \\
\hline
\end{tabular}

